\title{
EGRET excess of diffuse galactic gamma rays as tracer of dark matter ${ }^{\star}$
}

\author{
W. de Boer ${ }^{1}$, C. Sander ${ }^{1}$, V. Zhukov ${ }^{1, \star \star}$, A. V. Gladyshev ${ }^{2,3}$, and D. I. Kazakov²,3
}

\author{
${ }^{1}$ Institut für Experimentelle Kernphysik, Universität Karlsruhe (TH), PO Box 6980, 76128 Karlsruhe, Germany \\ e-mail: Wim.de.Boer@cern.ch; [deboer; sander; zhukov]@ekp.uni-karlsruhe.de \\ 2 Bogoliubov Laboratory of Theoretical Physics, Joint Institute for Nuclear Research, 141980 Dubna, Moscow Region, Russia \\ e-mail: [gladysh;kazakovd] @theor.jinr.ru \\ 3 Institute of Theoretical and Experimental Physics, 117218, 25 B. Cheremushkinskaya, Moscow, Russia
}

Received 29 June 2005 / Accepted 17 August 2005

\section{ABSTRACT}

The public data from the EGRET space telescope on diffuse Galactic gamma rays in the energy range from 0.1 to $10 \mathrm{GeV}$ are reanalyzed with the purpose of searching for signals of Dark Matter annihilation (DMA). The analysis confirms the previously observed excess for energies above $1 \mathrm{GeV}$ in comparison with the expectations from conventional Galactic models. In addition, the excess was found to show all the key features of a signal from Dark Matter Annihilation (DMA): a) the excess is observable in all sky directions and has the same shape everywhere, thus pointing to a common source; $b$ ) the shape corresponds to the expected spectrum of the annihilation of non-relativistic massive particles into - among others - neutral $\pi^{0}$ mesons, which decay into photons. From the energy spectrum of the excess we deduce a WIMP mass between 50 and $100 \mathrm{GeV}$, while from the intensity of the excess in all sky directions the shape of the halo could be reconstructed. The DM halo is consistent with an almost spherical isothermal profile with substructure in the Galactic plane in the form of toroidal rings at 4 and $14 \mathrm{kpc}$ from the center. These rings lead to a peculiar shape of the rotation curve, in agreement with the data, which proves that the EGRET excess traces the Dark Matter.

Key words. gamma rays: observations - gamma rays: theory - cosmology: diffuse radiation - Galaxy: structure - Galaxy: halo cosmology: dark matter

\section{Introduction}

Cold Dark Matter (CDM) makes up 23\% of the energy of the Universe, as deduced from the WMAP measurements of the temperature anisotropies in the Cosmic Microwave Background, in combination with data on the Hubble expansion and the density fluctuations in the universe (Spergel et al. 2003). The nature of the CDM is unknown, but one of the most popular explanation for it is the neutralino, a stable neutral particle predicted by Supersymmetry (Ellis et al. 1984; Jungman et al. 1996). The neutralinos, usually denoted by $\chi$, are spin 1/2 Majorana particles, which can annihilate into pairs of Standard Model particles, but other candidates are possible as well. The only assumption needed for this analysis, is that the DM particles were produced in thermal equilibrium with all other particles in the early Universe and its number density $n_{\chi}$ decreases from the high value in the early universe to the present low number density by annihilation, as it happened with the number density of baryons as well (Kolb \& Turner 1990). The relic density of CDM is inversely proportional to $\langle\sigma v\rangle$, the averaged annihilation cross section $\sigma$ of

\footnotetext{
* Appendix A is only available in electronic form at http://www . edpsciences.org

$\star \star$ On leave from INPMSU, Moscow State University, Russia.
}

DM particles multiplied with their relative velocity $v$. This inverse proportionality is obvious, if one considers that a higher annihilation rate, given by $\langle\sigma v\rangle n_{\chi}$, would have reduced the relic density before freeze-out, i.e. the time, when the expansion rate of the Universe, given by the Hubble constant, became equal to or larger than the annihilation rate. The relation can be written as

$\Omega_{\chi} h^{2}=\frac{m_{\chi} n_{\chi}}{\rho_{c}} \approx\left(\frac{2 \times 10^{-27} \mathrm{~cm}^{3} \mathrm{~s}^{-1}}{\langle\sigma v\rangle}\right)$.

The denominator in the last part of this equation was found to be $30 \%$ smaller than the value calculated in Jungman et al. (1996). For the present value of $\Omega h^{2}=0.113 \pm 0.009$, as measured by WMAP (Spergel et al. 2003), the thermally averaged total cross section at the freeze-out temperature of $m_{\chi} / 22$ must have been around $2 \times 10^{-26} \mathrm{~cm}^{3} \mathrm{~s}^{-1}$. This is a cross section typical for weak interactions and explains why the DM does not cluster strongly in the center of galaxies, like the baryons do: the cross sections are simply too small to have large energy losses when falling towards the center. Therefore the DM particles are generically called WIMPs, Weakly Interacting Massive Particles. All possible enhancements of the annihilation rate from the clustering of DM (usually called boost factor) will be calculated with respect to this generic cross section, which 
basically only depends on the value of the Hubble constant. Note that $\langle\sigma v\rangle$ as calculated from Eq. (1), is independent of the WIMP mass (except for logarithmic corrections), as can be shown by a detailed calculation (Kolb \& Turner 1990).

The stable decay and fragmentation products from Dark Matter Annihilation (DMA) are neutrinos, photons, protons, antiprotons, electrons and positrons. From these, the protons and electrons disappear in the sea of many matter particles in the universe, but the photons and antimatter particles may be detectable above the background, generated by particle interactions. Such searches for indirect Dark Matter detection have been actively pursued, see e.g. the reviews by Bergström (2000) and Sumner (2002) or more recently by Bertone et al. (2005). References to earlier work can be found in these reviews.

Gamma rays have the advantage that they point back to the source and do not suffer energy losses, so they are the ideal candidates to trace the dark matter density. The charged components interact with Galactic matter and are deflected by the Galactic magnetic field, so they do not point back to the source.

A detailed distribution of gamma ray fluxes for energies between 0.03 and $10 \mathrm{GeV}$ was obtained by the Energetic Gamma Ray Emission Telescope EGRET, one of the four instruments on the Compton Gamma Ray Observatory CGRO, which collected data during nine years, from 1991 to 2000. The diffuse component shows a clear excess of about a factor two over the expected background from known nuclear interactions, inverse Compton scattering and bremsstrahlung. The excess was observed first by Hunter et al. (1997), while the most complete calculation of the background is encoded in the GALPROP program (Moskalenko et al. 1998; Strong et al. 2000). This excess was shown to possess all the key features from dark matter annihilation (DMA), as discussed at various conferences and workshops (De Boer et al. 2004; De Boer 2005a,b). By fitting only the shapes of the background and DMA signal the analysis becomes independent of the absolute normalization of the background. Therefore uncertainties in the background fluxes from e.g. the gas densities and cosmic ray fluxes are eliminated.

Apart from fitting the shapes of DMA signal and background the present analysis differs also from previous ones (see e.g. Berezinsky et al. 1992, 1994; Jungman \& Kamionkowski 1995; Bergström et al. 1998; Fargion et al. 1999; Bergström et al. 1999, 2001; Bertone et al. 2002; Tyler 2002; Ullio et al. 2002; Hooper \& Dingus 2002; Edsjo 2002; Cesarini et al. 2004; Morselli 2004; Aloisio et al. 2004; Tasitsiomi et al. 2004) by the fact that the fluxes and the energy spectrum in all sky directions were considered simultaneously. This requires a complete reanalysis of the publicly available EGRET data, since the diffuse gamma ray data have been published only in a limited number of sky directions.

Knowing the WIMP number density $n_{\chi}$ from the flux and the WIMP mass $m_{\chi}$ from the spectrum allows to reconstruct the shape of the DM mass $\left(=n_{\chi} \cdot m_{\chi}\right)$ distribution in the Galaxy, which in turn can be used - in combination with the distribution of visible matter - to reconstruct the shape of the rotation curve. The absolute normalization of the DM density distribution or halo profile can be obtained by requiring that the local rotation velocity of our solar system at $8.3 \mathrm{kpc}$ is $220 \mathrm{~km} \mathrm{~s}^{-1}$. It will be shown that the rotation curve, as obtained from the EGRET excess of gamma rays, provides the first explanation for the peculiar change of slope in the rotation curve at around $11 \mathrm{kpc}$, indicating that the excess traces DM.

The paper has been organized as follows: Sect. 2 explains the fitting method, Sect. 3 describes the analysis of the EGRET data, Sect. 4 describes the determination of the DM halo profile and comparison with the Galactic rotation curve, Sect. 5 discusses possible objections to the DMA interpretation of the EGRET excess and Sect. 6 summarizes the results.

\section{Fitting method for indirect dark matter annihilation}

As mentioned in the introduction, neutral particles play a very special role for indirect DM searches, since they point back to the source. Therefore the gamma rays provide a perfect means to reconstruct the halo profile of the DM by observing the intensity of the gamma ray emissions in the various sky directions.

Of course there are also many sources of diffuse gamma rays in the Galaxy, so disentangling the annihilation signal is at first glance not easy. However, the spectral shapes of the diffuse gamma ray backgrounds are well known from accelerator experiments for all processes: the dominant background from decays of $\pi^{0}$ mesons produced in nuclear interactions, contributions from inverse Compton scattering of electrons on photons and Bremsstrahlung from electrons in the Coulomb field of nuclei. The best estimate of the relative contributions is given by the GALPROP code (Strong \& Moskalenko 1998; Moskalenko et al. 1998; Strong et al. 2000), which parametrizes the gas densities, cross sections and energy spectra for all processes of interest and solves numerically the diffusion equation to obtain a complete solution for the density map of all primary and secondary nuclei. For this the so-called "conventional" GALPROP model was used, which assumes the spectra of protons and electrons, as measured locally in the solar system, to be representative for the whole Galaxy. For protons, which have negligible energy losses, this is a reasonable assumption; for electrons, which have larger energy losses from ionization and Bremsstrahlung, this assumption may be questioned. Therefore we have restricted the analysis to gamma ray energies above $0.07 \mathrm{GeV}$, in which case the $\pi^{0}$ component starts to become dominant: electron-induced gamma ray production is of the same order of magnitude as the nuclei-induced gamma ray production at this energy, but at $0.5 \mathrm{GeV}$ the electroninduced component is already below $10 \%$ for the inner Galaxy and below $25 \%$ for other regions. Therefore one is not too sensitive to electron-induced gamma rays in the region of the EGRET excess, which is maximal at energies around 2-4 GeV. The "conventional" model is to be contrasted with the "optimized" model (Strong et al. 2004), in which case the electron and proton spectra are "optimized" to explain the EGRET excess without DM by allowing them to deviate from the locally observed spectra. Even the freedom for both the proton and electron spectra does not lead to particular good fits of the background to the EGRET data, if all sky directions are considered, as will be discussed in the next section. 
As mentioned in the introduction, only the shape of the background is needed for the fit, not the absolute normalization. Leaving the normalization free in the fit for a given sky direction is important, simply because one does not know the cosmic ray and gas densities to better than about $20 \%$ for a given sky direction. On the other hand, one knows for given cosmic ray spectra the shape of the expected gamma rays quite well: for electron-induced gamma production these processes can be easily calculated, while for the nuclei-induced processes the gamma ray spectra are known from scattering of beams of nuclei on fixed targets. In the galaxy the "beams" are the cosmic rays, while the gas in the disk is the fixed target. The wealth of data on nuclear interactions have resulted in the so called string fragmentation model, encoded e.g. in the PYTHIA program from Sjöstrand et al. (2003), which describes gamma ray production from nuclear interactions well.

The small contribution from the electron-induced gamma rays was added to the dominant contribution from $\pi^{0}$ decays and the shape of the total background thus obtained was used for a given sky direction. The relative fraction from electroninduced and nuclei-induced gamma rays varies with sky direction and this fraction was taken from GALPROP, but a fit with a constant fraction yielded similar results for the DM profile. Given that we do not attempt to determine the absolute normalization, the analysis is not sensitive to the many GALPROP propagation parameters determining absolute density profiles of the Galactic components. Furthermore the propagation of the gamma rays is straightforward. The fitted normalization factor of the background in each direction was found to agree within errors with the values determined from the GALPROP code, as will be discussed later.

WIMPs are expected to annihilate into fermion-antifermion or gauge boson pairs, so a large fraction will result in quarkantiquark pairs, which produce typically $30-40$ photons per annihilation in the fragmentation process (mainly from $\pi^{0}$ decays). However, the photons from DMA are expected to have a spectrum significantly different from the ones from nuclear interactions. This can be understood qualitatively as follows: the WIMPs are strongly non-relativistic, so they annihilate almost at rest. Therefore DM annihilates into almost mono-energetic pairs of particles with an energy approximately equal to the WIMP mass. This results in a rather energetic gamma ray spectrum with a sharp cut-off at the WIMP mass. Such gamma ray spectra from the fragmentation of mono-energetic quark pairs have been measured precisely at electron-positron colliders and the data are well described by the string fragmentation model mentioned above. The expected gamma ray spectra from this program are shown in Fig. 1 for a WIMP mass of $100 \mathrm{GeV}$ and several annihilation channels. The difference between various channels is small, except for the leptonic final state, but this is expected to be suppressed. Such a gamma energy spectrum from DMA is considerably harder than the one from nuclear interactions, which can be calculated by PYTHIA as well. The difference in shapes allows one to fit to the data simultaneously the spectra from background and DMA, thus treating the absolute normalizations as free parameters, which is an advantage, since the shapes of the spectra are much better known than the absolute fluxes. The fitted value of the normalization factor of

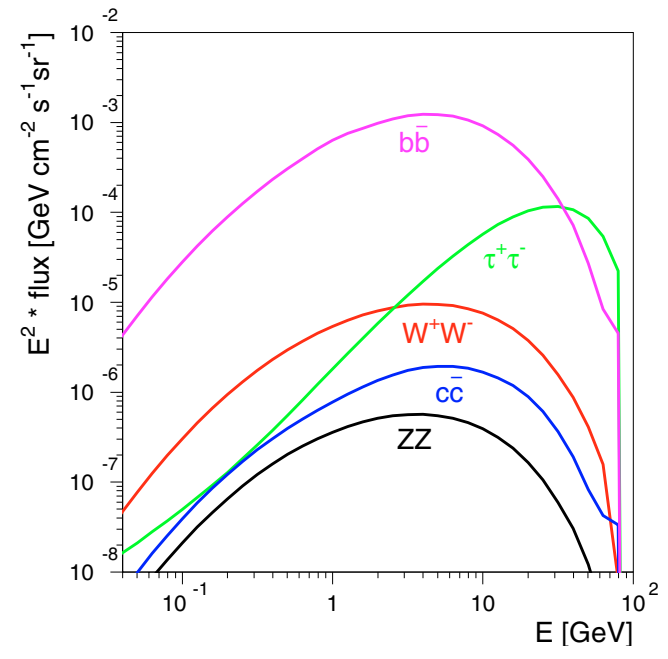

Fig. 1. The expected shape of gamma ray spectra for the fragmentation of WIMPs into different final states (arbitrary normalization): heavy quark pairs (b, c), heavy gauge bosons (W, Z) and $\tau$ leptons. The annihilation of neutralinos, the preferred WIMP candidates, into light final states is helicity suppressed (Jungman et al. 1996).

the DM component is sensitive to the DM clustering, which is expected in the form of clumps of DM with a mass spectrum starting may be as low as $10^{-6}$ solar masses (Berezinsky et al. 2004; Diemand et al. 2005). Such a clumpy structure is automatically generated in the hierarchical formation of galaxies. However, the DMA is higher in the clumps than outside, since the local density is there higher and DMA is proportional to the local density squared, i.e. $\left\langle\rho_{\mathrm{DM}}^{2}\right\rangle$. This can be considerably larger than $\left\langle\rho_{\mathrm{DM}}\right\rangle^{2}$ and the ratio is the enhancement or boost factor, i.e. $B=\left\langle\rho_{\mathrm{DM}}^{2}\right\rangle /\left\langle\rho_{\mathrm{DM}}\right\rangle^{2}$. The rotation curve is only sensitive to the total mass, i.e. $\left\langle\rho_{\mathrm{DM}}\right\rangle$. The boost factor can be obtained from the fitted normalization and can be large, from a few tens to a few thousands, depending on the unknown details of the DM clustering.

In addition to the Galactic background (GB) one expects a contribution from the extragalactic background (EGBG). The origin of these gamma rays can be other galaxies which may yield similar contributions as our Galaxy, or quite different sources like Active Galactic Nuclei (AGN), quasars or blazars. Since each extragalactic object has individual properties, it is difficult to predict the shape or the absolute value of this background component. Experimentally the EGBG can be obtained by subtracting from the EGRET data the Galactic contribution using the extrapolation method pioneered by Sreekumar et al. (1998). Of course, the Galactic contribution includes a contribution from Galactic DM, which is dependent on the EGBG, so the EGBG can only be obtained in an iterative procedure, as was done by Sander (2005). This contribution is taken to be of the same shape and same magnitude in all sky directions. It starts to become important towards the galactic poles, where both the galactic background and the DMA become small. 


\section{Analysis of EGRET data}

The EGRET data are publicly available as high resolution $(0.5 \times 0.5$ degree $)$ sky maps from the NASA archive ${ }^{1}$, which allows an analysis in many independent sky directions after convolution with the point-spread function, which is a function of energy and becomes important for gamma rays below $1 \mathrm{GeV}$. The data set with the known point sources subtracted have been used. The point sources are defined by a $5 \sigma$ enhancement above the diffuse background. In general these point sources are only a small fraction of the total gamma ray flux, so the analysis is not sensitive to the subtraction of point sources. There are only a few nearby strong sources, which dominate the flux in these directions and the subtraction causes an additional systematic uncertainty. Therefore these directions have been excluded in the determination of the halo profile, which requires a fine scanning of all sky directions, as will be discussed in the next section. The contribution of the subtracted point sources have been indicated in the spectra for 180 independent sky directions, shown in the Appendix.

The EGRET telescope was carefully calibrated at SLAC with a quasi mono-energetic photon beam in the energy range of 0.02 to $10 \mathrm{GeV}$ (Thompson et al. 1987). The efficiency and calibration during flight was also carefully monitored (Esposito et al. 1999). Using Monte Carlo simulations the energy range was recently extended up to higher energies with a correspondingly larger uncertainty, mainly from the self-vetoing of the detector by the back-scattering from the electromagnetic calorimeter into the veto counters for high energetic showers (Strong et al. 2004; Thompson et al. 2005). Due to this uncertainty only data below $10 \mathrm{GeV}$ were used in the fits discussed below. In total 8 energy ranges were used: $0.10-0.15 \mathrm{GeV}$; 0.15-0.30 GeV; 0.30-0.50 GeV, 0.5-1.0 GeV; 1.0-2.0 GeV; 2.0-4.0 GeV; 4.0-10.0 GeV. The data points have been plotted at the arithmetic mean of the low and high endpoints of the bin, i.e. at $\sqrt{E_{\text {low }} E_{\text {high }}}$.

With the 9 years of data taking with the EGRET telescope 180 independent sky directions can be studied without statistical problems. However, the data is limited by systematic uncertainties, which have to be taken into account carefully. The overall normalization error is usually quoted as $15 \%$, but the relative point-to-point error is much smaller. The latter can be determined by fitting the energy spectrum with a polynomial and if a certain energy bin is left out of the fit, then its variance with respect to the other energy points can be determined to be between 3 and $7 \%$. Therefore, if one fits only the shape of the spectrum with a free normalization parameter, only these relative errors between the energy points are the relevant ones, which were taken to be $7 \%$.

Fitting the known shapes of the three contributions (GB, EGBG, DMA) to the EGRET data, as discussed before in Sect. 2, yielded astonishingly good fits, as shown in Fig. 2 for the 6 different sky directions given in Table 1. For energies between 0.07 and $0.5 \mathrm{GeV}$ the flux is dominated by the background, while above these energies a clear contribution from Dark Matter annihilation is needed. The excess in

\footnotetext{
${ }^{1}$ NASA archive: http://cossc.gsfc.nasa.gov/archive/
}

different sky directions can be explained by a single WIMP mass around $60 \mathrm{GeV}$ and a single boost factor of about 100 . The free normalization of the background comes out to be in reasonable agreement with the absolute predictions from the GALPROP propagation model of our Galaxy (Strong \& Moskalenko 1998; Moskalenko et al. 1998; Strong et al. 2000), as shown in Fig. 3 for a fine binning of the skymaps. Thus the fitting method yields the correct normalization for the BG and the high energy excess for a given background shape determines the normalization for the DMA. The excess in all sky directions has a similar shape, as demonstrated in Fig. 4 for the first five sky regions of Table 1 . The quality of the EGRET data can be appreciated from Fig. 4, where only the statistical errors are plotted. They are only visible at large latitudes. The right hand side of Fig. 4 shows the plots for WIMP masses of 50 and $70 \mathrm{GeV}$; the $70 \mathrm{GeV}$ WIMP mass clearly fits worse. WIMP masses below $50 \mathrm{GeV}$ lead to a too low relic density, since in that case one hits the $Z^{0}$-resonance and for WIMP masses below $40 \mathrm{GeV}$, i.e. on the other side of the resonance, the fit to the EGRET becomes worse. Therefore a lower limit of $50 \mathrm{GeV}$ is taken and the $95 \%$ C.L. upper limit depends somewhat on the background model: $70 \mathrm{GeV}$ for the shape of the conventional model and more like $100 \mathrm{GeV}$ for the shape of the optimized model. Therefore the WIMP mass is estimated to be between 50 and $100 \mathrm{GeV}$.

Alternative explanations for the excess have been plentiful. Among them: locally soft electron and proton spectra, implying that in other regions of the Galaxy the spectra are harder, thus producing harder gamma ray spectra. A summary of these discussions has been given by Strong et al. (2004), who find that hard proton spectra are incompatible with the antiproton yield and hard electron spectra are difficult to reconcile with the EGRET data up to $120 \mathrm{GeV}$. However, they find that by modifying the electron and proton injection spectra simultaneously, the description of the data can be improved by increasing the fluxes at high energies. The energy dependence at high energies is kept with the same slope as the locally measured spectra, which is required at least for protons, since the energy loss time of protons above a few $\mathrm{GeV}$ is of the order of the lifetime of the universe. Therefore it is hard to have strong inhomogeneities in the proton spectra at high energies. At low energies the fluxes are reduced by solar modulations, so here the spectral shapes of protons and electrons have large uncertainties and the shape can be optimized to fit the EGRET data.

The problem with this "optimized solution" is however that the shape of the gamma spectra is improved but still not reproduced well, as shown in Fig. 5 for the regions of Table 1. But it is exactly the shape, which was well measured by EGRET, because the relative errors between neighbouring energies are roughly half of the normalization error of $15 \%$. The probability, as calculated from the total $\chi^{2} /$ d.o.f. $=110 / 42$, as indicated for each region in Fig. 5, is below $10^{-7}$. The fact that the shape is not well fitted in the optimized model can also be seen from Fig. 9 in the original publication (Strong et al. 2004), which shows the longitudinal profile for various energy bins: above $1 \mathrm{GeV}$ the prediction of the model is clearly too low, especially if one takes into account that the statistical errors in the Galactic plane are negligible, so the plotted errors of $15 \%$ are 

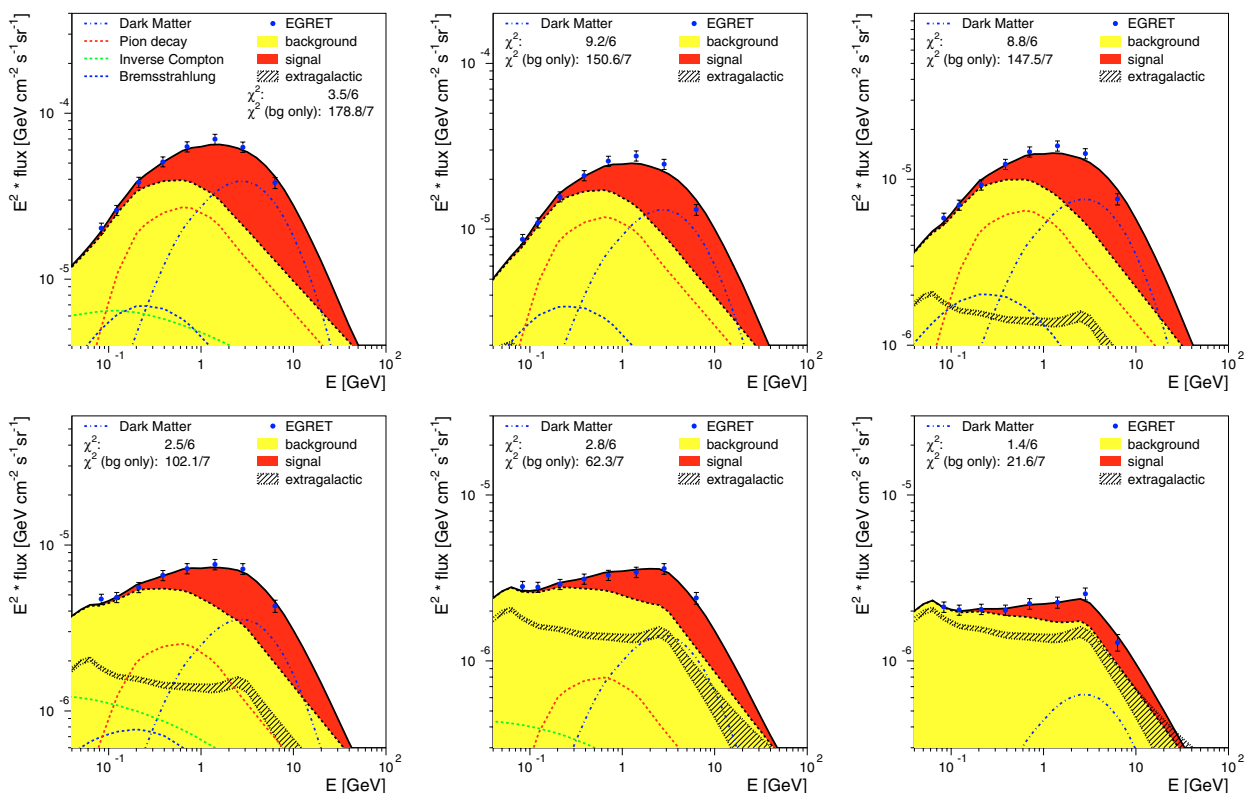

Fig. 2. Fit of the shapes of background and DMA signal to the EGRET data in the Galactic disk (top row, regions A, B, C from Table 1) and outside the Galactic disk (bottom row, regions D, E, F). The light shaded (yellow) areas indicate the background using the shape of the conventional GALPROP model, while the dark shaded (red) areas are the signal contribution from DMA for a 60 GeV WIMP mass. The individual shapes of background and DMA have been indicated by dashed lines, while the extragalactic background is given by the hatched area. The $\chi^{2}$ of the background is determined with an independent background only fit, which yields a probability practically zero, as can be estimated from the indicated $\chi^{2}$ values for the statistically independent regions. The fit including DM has a total probability around 0.8 .

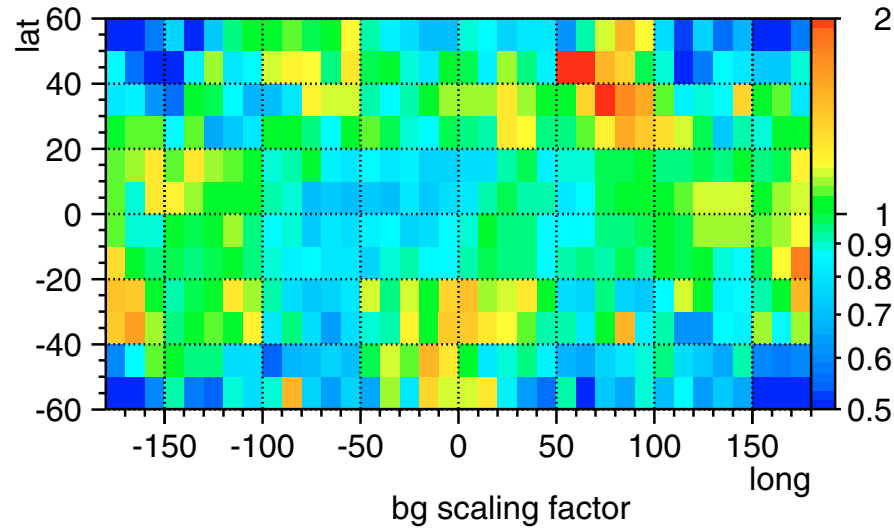

Fig. 3. The ratio of the fitted background normalization and the absolute prediction of the conventional GALPROP model (Strong et al. 2004) as function of latitude and longitude. For this plot a fine binning was used $(90 \times 45)$; for the whole sky the scaling is around 1, i.e. the background determination with our method is in good agreement with GALPROP, except for the disk region with latitudes below 50, where a systematic deviation of $20-30 \%$ is seen. Since the density in the disk is known to be asymmetric, but GALPROP uses a symmetric parametrization, this discrepancy is understandable.

correlated. And this discrepancy above $1 \mathrm{GeV}$ is observed in all directions, but this energy range is exactly where DMA contributes. Adding DM to the optimized model improves the fit probability from below $10^{-7}$ to 0.8 , as shown in Fig. 6. Of course, the DM contribution is smaller than in case of the conventional background in Fig. 2, which results in a reduction of the boost factor by roughly a factor three. Similar results are obtained for the shape proposed by Kamae et al. (2005). As with
Table 1. The six sky regions mentioned in the text; the detailed halo profile was obtained from 180 independent sky directions, as described in the Appendix.

\begin{tabular}{cccc}
\hline \hline Region & Longitude $l$ & Latitude $|b|$ & Description \\
\hline A & $330-30$ & $0-5$ & Inner Galaxy \\
B & $30-330$ & $0-5$ & Disk without inner Galaxy \\
C & $90-270$ & $0-10$ & Outer Galaxy \\
D & $0-360$ & $10-20$ & Low latitude \\
E & $0-360$ & $20-60$ & Intermediate latitude \\
F & $0-360$ & $60-90$ & Galactic Poles \\
\hline
\end{tabular}

the optimized model, the absolute prediction of Kamae et al. (2005) overshoots the low energy data and undershoots the high energy data, so if only the shape is fitted with a free normalization factor, the excess is clearly present. Their proposed contribution of diffractive pp-scattering only reduces the excess by $10-20 \%$ and if in addition the proposed harder proton spectrum is used (spectral index -2.5 instead of -2.7 measured locally), the excess can be reduced by $30 \%$ In all cases the proposed background shape from Kamae et al. (2005) fits the data in the different regions considerably worse than the optimized model from Strong et al. (2004), mainly because Kamae et al. (2005) try to improve the fit by changing the proton spectrum only, while in the optimized model both the electron spectrum and proton spectrum are modified. 

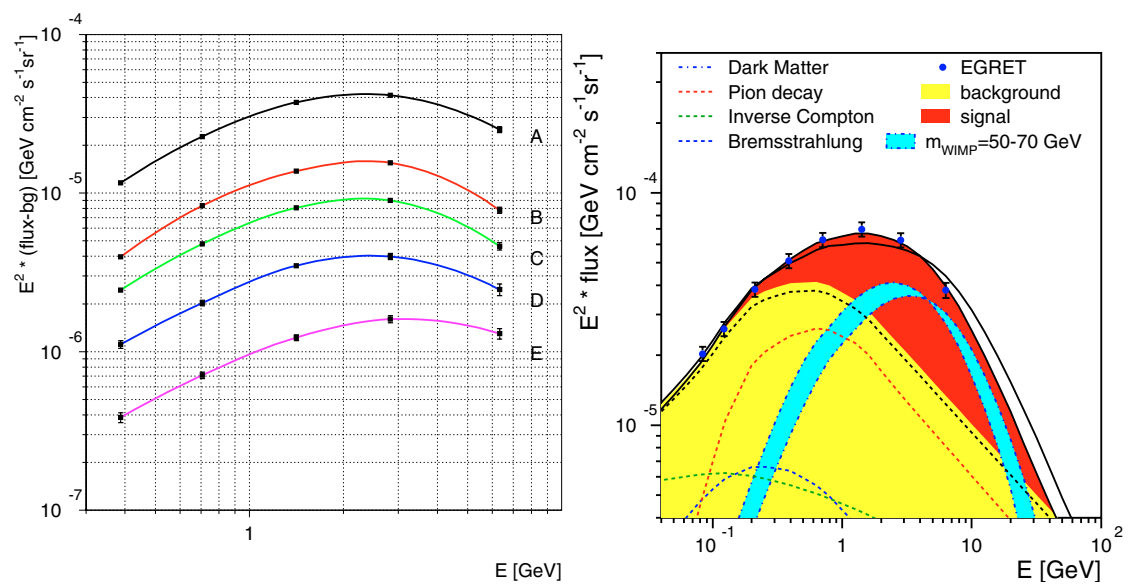

Fig. 4. Left: the difference of the observed EGRET flux and fitted background for the various regions of Table 1, i.e. the red area in Fig. 2. One observes the same spectral shape for all regions, indicating a common source for the excess. Only the statistical errors have been plotted and the curves are fitted spline functions to guide the eye. Right: the influence of the WIMP mass on the spectrum: the light shaded (blue) curve shows the influence of a WIMP mass variation between 50 and $70 \mathrm{GeV}$. The lower (upper) solid line at the highest energies corresponds to the total flux for a 50 (70) GeV WIMP mass.
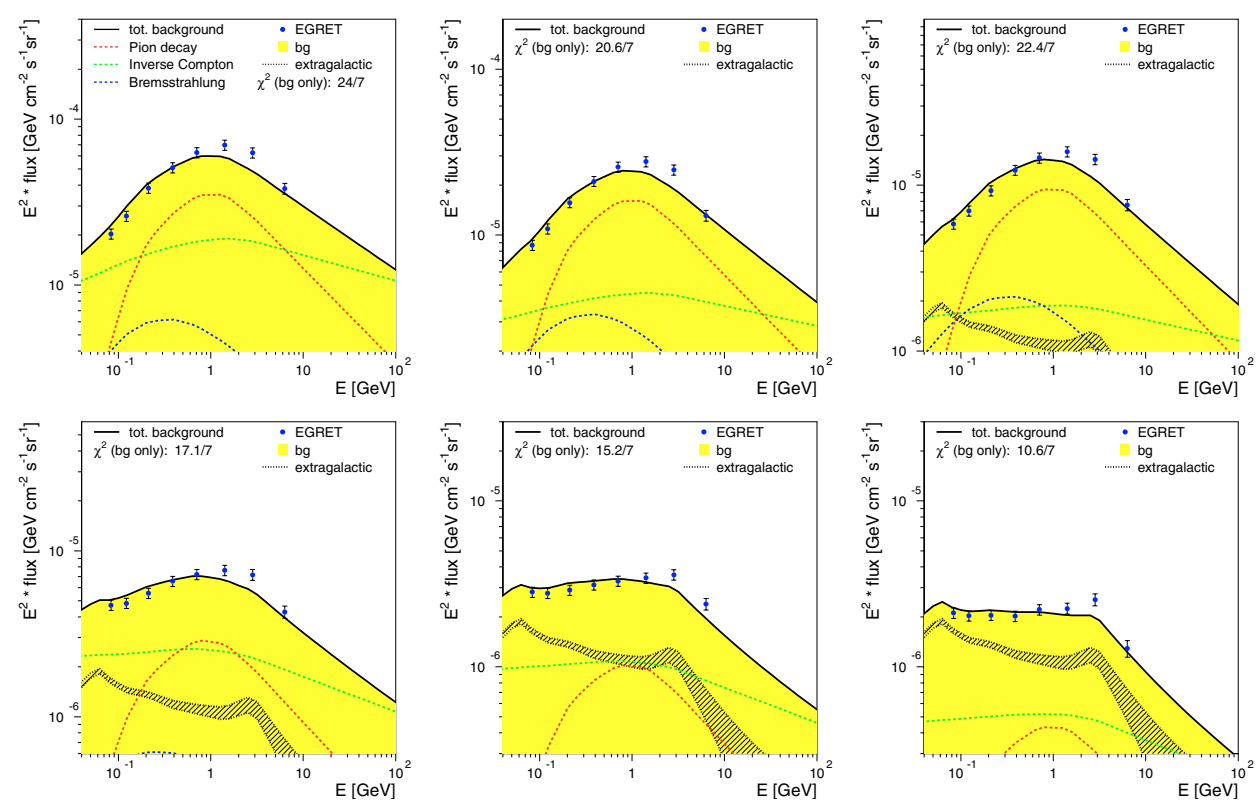

Fig. 5. Fit results of the shape of the optimized model to the EGRET data; regions and coding as for Fig. 2, but without DMA contribution. The excess above $1 \mathrm{GeV}$ can be improved by adding a DM contribution, in which case the normalization of the background (solid line) will become lower again and fit the low energy data points.

\section{Determination of the halo profile}

From the excess in the various sky directions one can obtain the halo profile. However, this requires a finer sampling of the various sky directions than discussed above. Therefore the fits to the 6 regions were repeated for the following regions: the longitude distributions are split into bins of $8^{\circ}$ for four different latitude ranges (absolute values of latitude: $0-5^{\circ}, 5-10^{\circ}$, $10-20^{\circ}, 20-90^{\circ}$ ), so one has $4 \times 45=180$ independent sky regions. The fit result for each of these regions has been displayed in the Appendix. Also the fluxes from the point sources are displayed, which have been subtracted from the data. In most regions the point sources have a negligible contribution except for some disk regions with strong pulsars (CRAB at $l \approx-175^{\circ}$, GEMINGA at $l \approx-165^{\circ}$, VELA at $l \approx-98^{\circ}$ and CYGNUS at $l \sim+80^{\circ}$ ). These regions have been left out of the fit for the halo parameters.

The differential gamma flux in a direction forming an angle $\psi$ with the direction of the Galactic center is given by:

$$
\phi_{\chi}(E, \psi)=\frac{\langle\sigma v\rangle}{4 \pi} \sum_{f} \frac{\mathrm{d} N_{f}}{\mathrm{~d} E} b_{f} \int_{\text {line of sight }} B_{l} \frac{1}{2} \frac{\left\langle\rho_{\chi}\right\rangle^{2}}{M_{\chi}^{2}} \mathrm{~d} l_{\psi}
$$

where $b_{f}$ is the branching ratio into the tree-level annihilation final state, while $\mathrm{d} N_{f} / \mathrm{d} E$ is the differential photon yield for the final state $f$. The spectrum $\mathrm{d} N_{f} / \mathrm{d} E$ is similar for all hadronic final states, as discussed before in Sect. 2. The cross section $\langle\sigma v\rangle$ 

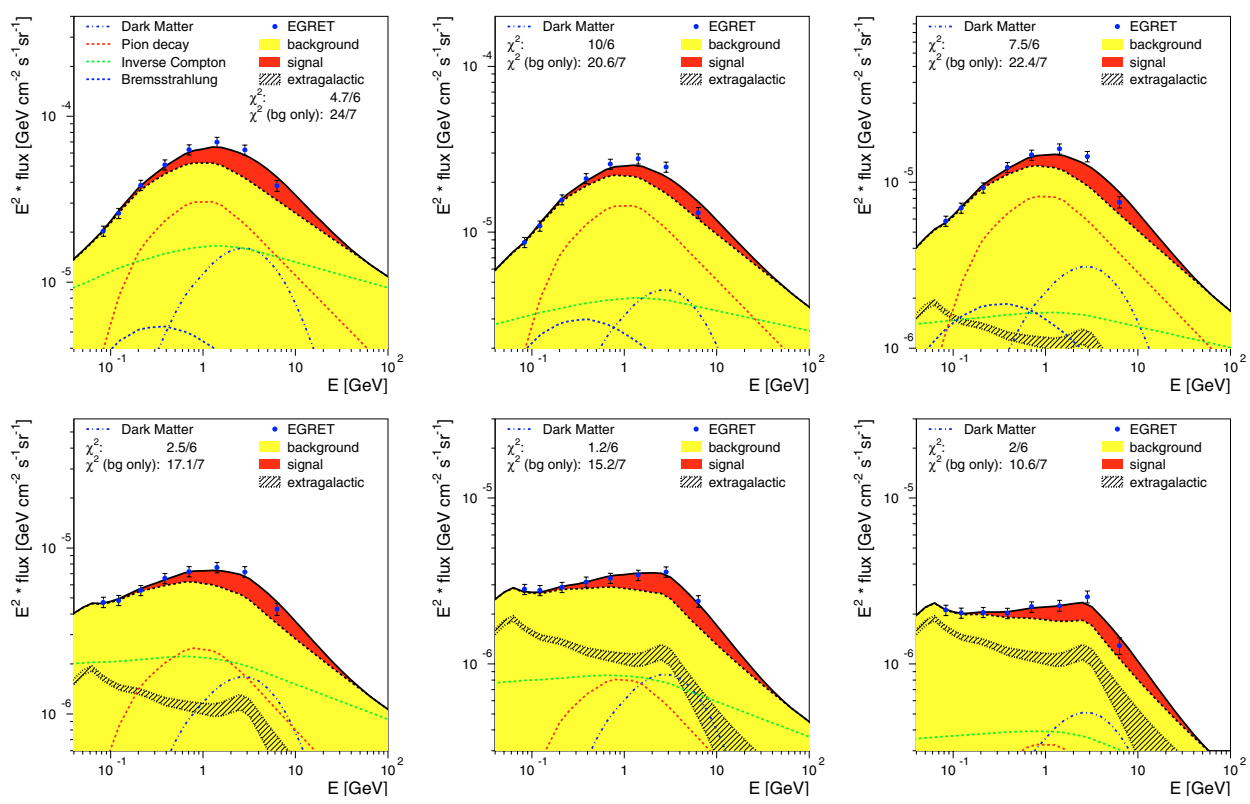

Fig. 6. Fit results of the shape of the optimized model plus DMA to the EGRET data; region and coding as for Fig. 2. The fit is now equally good as with the conventional background shape in Fig. 2, but the boost factor is roughly a factor three lower.

is taken from Eq. (1) with the WMAP value for $\Omega h^{2}$ (Spergel et al. 2003). So one observes from this equation, that for a given excess and a known WIMP mass (around $60 \mathrm{GeV}$ from the spectrum of the excess as discussed above), the only unknown quantity in the direction $\psi$ is the square of the averaged WIMP mass density $\rho_{\chi}$ multiplied by the boost factor $B_{l}=\left\langle\rho_{\chi}^{2}\right\rangle /\left\langle\rho_{\chi}\right\rangle^{2}$, which represents the enhancement of the annihilation rate by the clustering of DM. If one assumes that the clustering is similar in all directions, i.e. the same boost factors in all directions, the DM density profile $\rho(r)$ can be determined from the excess in the various directions. The most transparent way to do this is the following: search for a functional form of the profile as function of the distance from the Galactic center and see which form yields the best fit. A survey of the optical rotation curves of 400 galaxies shows that the halo distributions of most of them can be fitted either with the NavarroFrenk-White (NFW) or the pseudo-isothermal profile or both (Jimenez et al. 2003). These halo profiles can be parametrized as follows:

$\rho(r)=\rho_{0} \cdot\left(\frac{r}{a}\right)^{-\gamma}\left[1+\left(\frac{r}{a}\right)^{\alpha}\right]^{\frac{\gamma-\beta}{\alpha}}$,

where $a$ is a scale radius and the slopes $\alpha, \beta$ and $\gamma$ can be roughly thought of as the radial dependence at $r \approx a, r \gg a$ and $r \ll a$, respectively. The cuspy NFW profile (Navarro et al. $1996)$ is defined by $(\alpha, \beta, \gamma)=(1,3,1)$ for a scale $a=10 \mathrm{kpc}$, while the Moore profile with $\gamma=1.2$ is even more cuspy (Diemand et al. 2005). The isothermal profile with $(\alpha, \beta, \gamma)=$ $(2,2,0)$ has no cusp $(\gamma=0)$, but a core which is taken to be the size of the inner Galaxy, i.e. $a=5 \mathrm{kpc}$ and $\beta=2$ implies a flat rotation curve. The EGRET excess towards the Galactic center does not show a cusp, but is perfectly flat near the center as expected for a cored profile, so a cored isothermal profile was fitted to the excess in 180 sky directions.
In general, the boost factor towards the Galactic center may be lower than in other directions because of the likely tidal disruption of small DM clusters by the fly-by from stars. In this case the flux is proportional to $B(r)\left\langle\rho(r)^{n}\right\rangle$, where $n$ can vary between 1 and 2 depending on the DM clustering: $n=2$ if no clustering and $n=1$ if DMA is predominantly in non-overlapping clusters. Consequently one has many alternatives to fit, which are outside the scope of the present paper. Therefore we concentrate on a boost factor independent of $r$ and $n=2$, which turns out to yield a good fit.

The fit results are shown in Fig. 7 for gamma energies below $0.5 \mathrm{GeV}$ and in Fig. 8 for gamma energies above $0.5 \mathrm{GeV}$ as function of longitude for various latitudes, i.e. one determines the flux towards the earth by looking around in a full circle either in the Galactic plane or at various angles above and below the disk. Clearly, the data are well described in all directions for data below $0.5 \mathrm{GeV}$ with hardly any contribution from DMA, while for data above $0.5 \mathrm{GeV}$ only the data towards the Galactic poles are reasonably well described by the background plus DM component (dark (red) contribution). Towards the Galactic center and Galactic anticenter the isothermal profile does not provide enough DM, as is obvious in the upper panels of Fig. 8. There are several reasons why this might be so. For example the infall of a satellite galaxy into the gravitational potential of larger galaxy can lead to toroidal ringlike DM overdensities (Hayashi et al. 2003). When ringlike structures were added with the radius and widths of the ring in and out of the plane as free parameters, the fit quickly converged for only two toroidal ringlike structures, namely at radii of 4 and $14 \mathrm{kpc}$. The enhanced gamma radiation at $14 \mathrm{kpc}$ was already observed in the original discovery paper of the excess (Hunter et al. 1997) and called "cosmic enhancement factor". Note that the radius of the ring can be determined from the longitude profile in the plane of the Galaxy, i.e. at low latitudes, because the solar system is not at the center, so if the density 

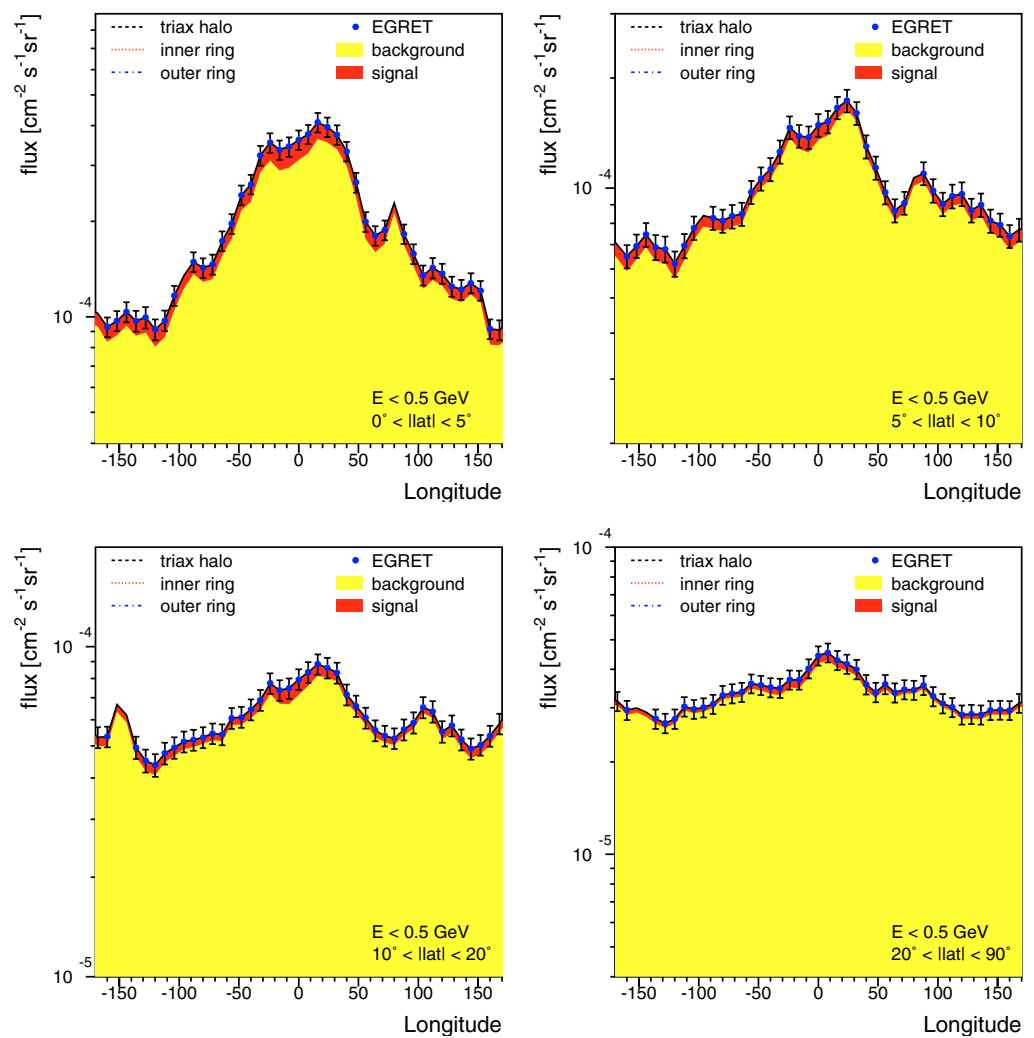

Fig. 7. The longitude distribution of diffuse Galactic gamma rays with energies below $0.5 \mathrm{GeV}$ for different latitudes The points represent the EGRET data. The contributions from the background and the almost negligible DMA for energies below $0.5 \mathrm{GeV}$ have been indicated by the light (yellow) and dark (red) shaded areas, respectively. The free normalization of the background for each bin provides a perfect description of the low energy data.
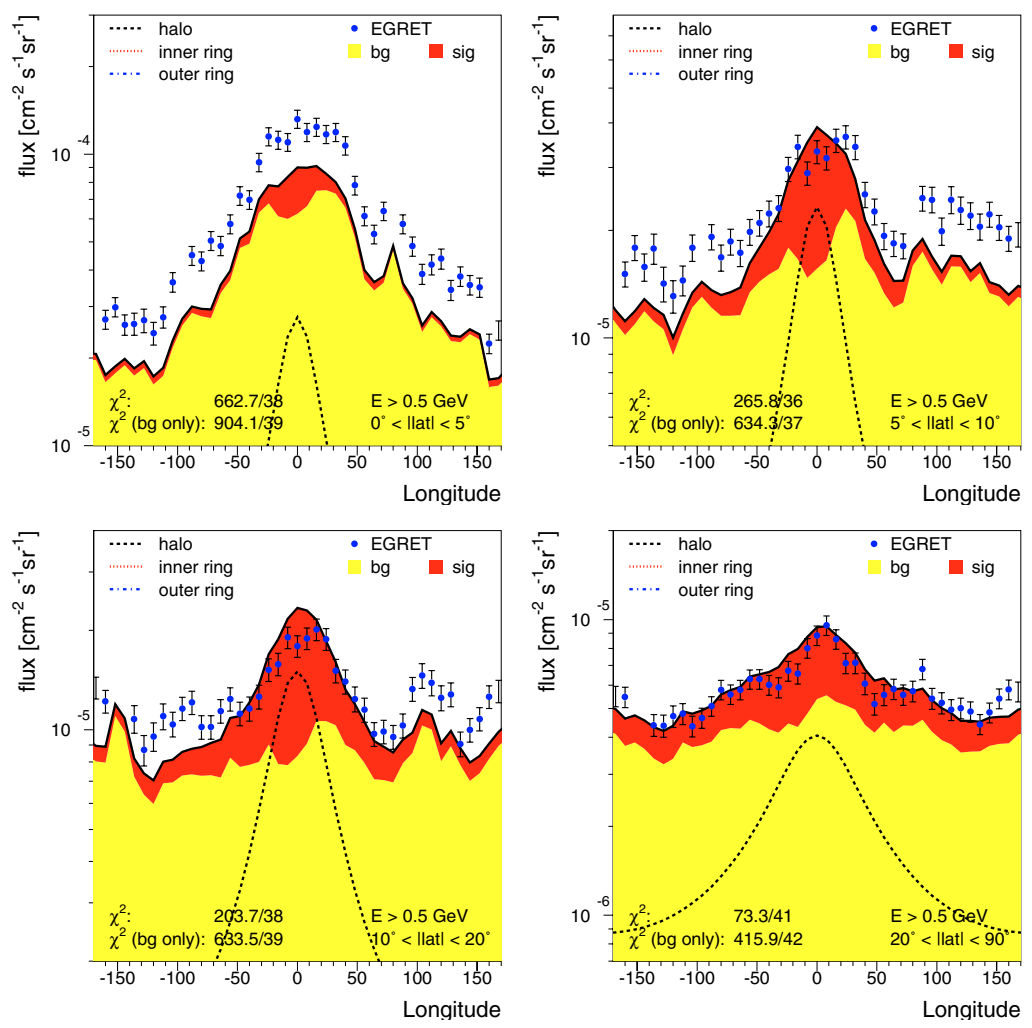

Fig. 8. As in Fig. 7, but for for EGRET data above $0.5 \mathrm{GeV}$ and DMA for an isothermal DM profile without ringlike substructures. 
is constant along the ring, different segments of the ring yield different fluxes which depend on the radius, orientation and ellipticity of the ring. The extent of the ring above the plane can be obtained from the longitude distribution for higher latitudes. It should be noted that the assumption of a constant density along the ring is not necessarily true. However, the fit is not very sensitive towards the ring density on the opposite side of the galactic center, so only the ring segment nearest to the solar system is assumed to have a constant density; this assumption yields a reasonable fit. Although the overdensities from the infall of a satellite galaxy do not produce rings, but at most ringlike segments during each passage near the pericenter, the precession of the orbit can lead to various segments along the pericenter. Since the analysis prefers a non-zero density over an angular range close to 360 degrees we continue to speak of "rings" of DM, although this does not mean at all perfect circularity.

With the rings the total DM halo profile can be parametrized as:

$$
\begin{aligned}
\rho_{\chi}(\boldsymbol{r})= & \rho_{0} \cdot\left(\frac{\tilde{r}}{r_{0}}\right)^{-\gamma}\left[\frac{1+\left(\frac{\tilde{r}}{a}\right)^{\alpha}}{1+\left(\frac{r_{0}}{a}\right)^{\alpha}}\right]^{\frac{\gamma-\beta}{\alpha}} \\
& +\sum_{n=1}^{2} \rho_{n} \exp \left(-\frac{\left(\tilde{r}_{g c, n}-R_{n}\right)^{2}}{2 \cdot \sigma_{R, n}^{2}}-\left|\frac{z}{\sigma_{z, n}}\right|\right)
\end{aligned}
$$

with

$$
\tilde{r}=\sqrt{x^{2}+\frac{y^{2}}{\varepsilon_{x y}^{2}}+\frac{z^{2}}{\varepsilon_{z}^{2}}}, \quad \tilde{r}_{g c, n}=\sqrt{x_{(n)}^{2}+\frac{y_{(n)}^{2}}{\varepsilon_{x y, n}^{2}}} ;
$$

$\varepsilon_{x y}$ and $\varepsilon_{z}\left(\varepsilon_{x y, n}\right)$ are the ellipticities of the triaxial halo profile and rings, respectively. The first term of Eq. (4) has been modified with respect to Eq. (3) in order that $\rho_{0}$ represents the DM density in the solar system, i.e. at $r=r_{0} \rho=\rho_{0}$. Other degrees of freedom are the angles with respect to the axis earth - Galactic center of the halo $\phi_{g c}$ and of the rings $\phi_{n}$, i.e. each component has its own coordinate system which is rotated around the $z$-axis. The maximum WIMP density of a ring $\rho_{n}$ is reached in the Galactic plane $(z=0)$ at a distance from the Galactic center $\tilde{r}_{g c, n}=R_{n}$. Figure 9 shows a schematic picture of a ring with the different definitions.

The radial width of the outer ring is taken to be different for the inner and outer side as can happen for the infall of a satellite galaxy, which is disrupted most strongly near the pericenter and the matter will be distributed towards larger distances, so the shape was modified to fall off to zero at radii smaller than the pericenter within a distance $d_{r}$ using two quadratic functions: $\rho(r)=a \cdot\left(r-\left(R_{n}-d_{r}\right)\right)^{2}$ for $\left(R_{n}-d_{r}\right)<r<\left(R_{n}-d_{r} / 2\right)$ and $\rho(r)=\rho_{n}-a \cdot\left(r-R_{n}\right)^{2}$ for $\left(R_{n}-d_{r} / 2\right)<r<R_{n}$.

The parameters of the halo model and the boost-factor are varied to minimize the following $\chi^{2}$ function:

$\chi^{2}=\sum_{i, j}\left(\frac{f^{i, j} \cdot \phi_{\mathrm{bg}}^{i, j}+B \cdot \phi_{\mathrm{dm}}^{i, j}+\phi_{\mathrm{eg}}-\phi_{\mathrm{exp}}^{i, j}}{\sigma^{i, j}}\right)^{2}$,

where $i$ and $j$ denote the different bins in longitude and latitude and $f^{i, j}$ and $B$ are the normalization factors of the

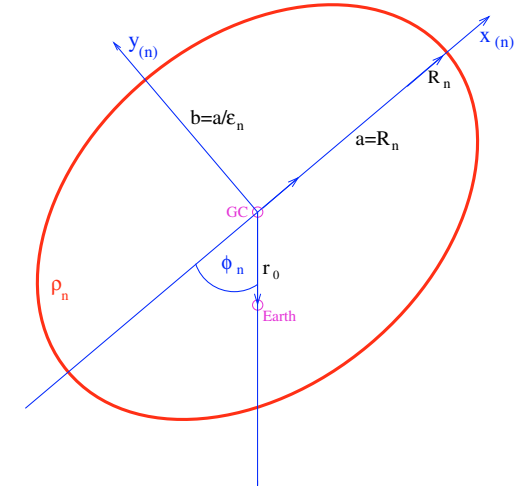

Fig. 9. Schematic picture of a DM ring with an elliptical shape and constant DM density $\rho_{n}$ along the ring. The definitions are also valid for the triaxial halo component, if the coordinate system is rotated with the appropriate angle $\phi_{n}$ towards the Galactic center and the short axis $c$ is modified by the ellipticity in the $z$-direction: $c=a / \epsilon_{z}$.

background and DMA. Note that the boost factor $B$ and the extragalactic flux $\phi_{e g}$ were taken to be the same in all directions, i.e. independent of $i$ and $j$. The scaling factor $\rho_{0}$ of the "spherical" component (=isothermal profile) is not a free parameter, since it is scaled to get the rotation velocity at $r_{0}$ correct. This means that if the fit requires a more massive inner ring, the density of the spherical component is adjusted automatically. Note that in total one fits 180 independent sky directions, each with 8 data points above $0.07 \mathrm{GeV}$, so one has a total of more than 1400 data points, which is enough to determine the halo parameters. In addition, the parameters of the different contributions are determined by completely independent data: the outer (inner) ring is determined by the flux in the plane of the disk away (towards) the Galactic center, while the isothermal profile is mainly determined by the fluxes outside the Galactic disk. As a test of the convergence and angular resolution a fit with 360 sky directions was performed as well, which yielded practically identical results.

All sky directions are now well described by the basic isothermal profile plus the substructure in the form of two toroidal rings, as shown in Fig. 10 with the contributions of the two rings indicated separately. They clearly dominate for low latitudes, but are small for latitudes above 10 degrees. The latitude distributions are also well described, as shown in Fig. 11 for the direction towards the Galactic center. The fit results of the parameters are summarized in Table 2 . The errors in the parameters are mainly systematic, e.g. depending on the fact that we took the boost factor to be the same in all directions etc. Determining these systematic errors is outside the scope of the present paper, but the qualitative picture of two ringlike substructures is independent of such details.

The boost factor for the profile with rings is around 100, if one assumes the DM annihilation cross section at the WIMP decoupling temperature $m_{\chi} / 22 \approx 3 \mathrm{GeV}$ in the early universe to be still valid for the low kinetic energies in the present universe. This is true, if the annihilation proceeds via the exchange of spin-less particles, like Higgs bosons. But the annihilation depends strongly on momenta for DMA via the exchange of a particle with spin, like the $Z$-boson. The resonant 

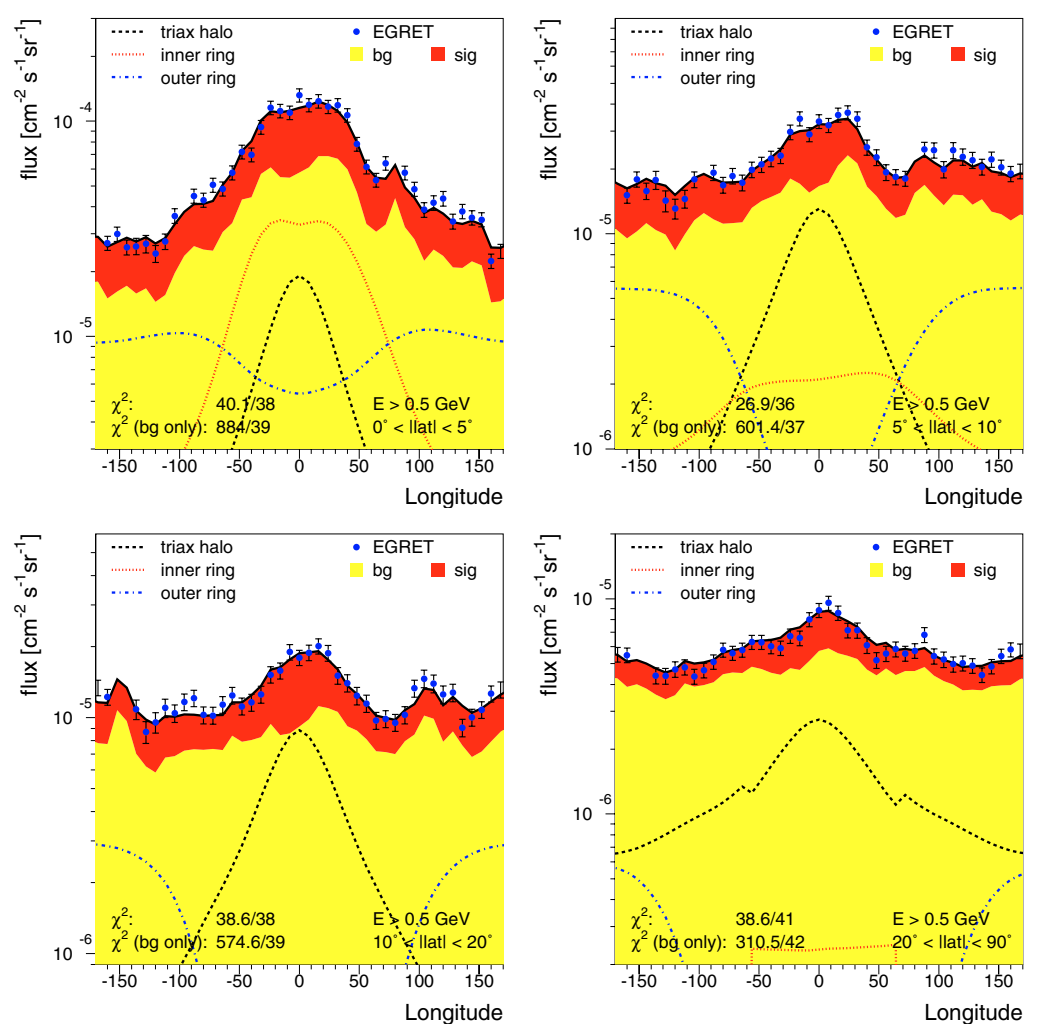

Fig. 10. As in Fig. 8, but including DMA for an isothermal DM profile with ringlike substructures at 4 and $14 \mathrm{kpc}$.
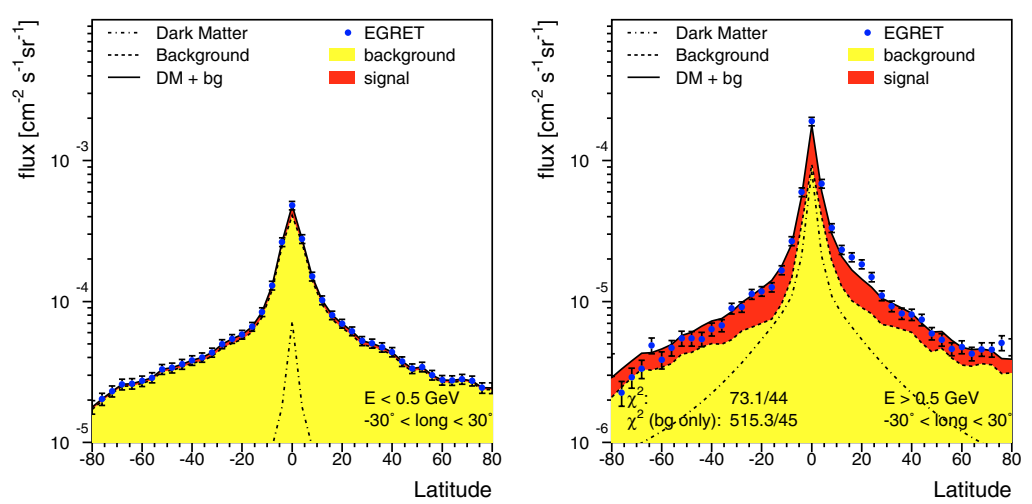

Fig. 11. The latitude distribution of diffuse gamma-rays for longitudes $-30^{\circ}<l<30^{\circ}$ and two energy bins: $E_{\gamma}<0.5 \mathrm{GeV}$ (left) and $E_{\gamma}>0.5 \mathrm{GeV}($ right). The points represent the EGRET data. The contributions from the background and the neutralino annihilation signal have been indicated by the light (yellow) and dark (red) shaded area, respectively. The fitted normalization factor of the background comes out in reasonable agreement with the GALPROP prediction, while the fitted normalization of the DM contribution corresponds to a WIMP mass around $60 \mathrm{GeV}$ with a boost factor of about 100 .

$Z$-exchange becomes dominant if the WIMP mass is close to half the $Z$-boson (around $45 \mathrm{GeV}$ ) and then the annihilation cross section at the temperatures of the present universe is much smaller, thus requiring boost factors of $10^{3}$ or more for WIMP masses below $55 \mathrm{GeV}$. Unfortunately, such large boost factors are not necessarily excluded, so one cannot determine a lower limit on the WIMP mass from the boost factors alone.

\subsection{Ring structure}

Figure 12 displays the halo distribution in the disk ( $x y$-plane) and perpendicular to the plane ( $x z$-plane) in a $3 \mathrm{D}$ plot, while Fig. 13 shows it in the projections in comparison with the distribution of baryonic matter. The contributions from the inner and outer rings at radii of 4.2 and $14 \mathrm{kpc}$, respectively can be clearly seen. The maximum ring densities of the inner (outer) ring are about a factor of 6 (7) higher than the isothermal profile at these maxima.

The maximum density of the outer ring is at a radius of $14 \mathrm{kpc}$ with a width of about $3.3 \mathrm{kpc}$ in radius and $1.7 \mathrm{kpc}$ perpendicular to the disk. These coordinates coincide with the ring of stars observed in the plane of the Galaxy at a distance of 14-18 kpc from the Galactic center (Newberg et al. 2002; Ibata et al. 2003; Yanni et al. 2003; Crane et al. 2003). These stars show a much smaller velocity dispersion $\left(10-30 \mathrm{~km} \mathrm{~s}^{-1}\right)$ and a larger $z$-distribution than the thick disk, so the ring 
Table 2. Fit results with and without the ringlike substructures. The triaxial halo and the two ring parameters with subscript 1 and 2 are given. The parameters $r_{0}, \alpha, \beta, \gamma, a_{0}$ are fixed by the definition of the pseudo-isothermal profile and $M_{200}, R_{200}, M_{1}, M_{2}$ are derived values, so the remaining 14 values have been optimized.

\begin{tabular}{|c|c|c|}
\hline parameter & halo without rings & halo with rings \\
\hline$\rho_{0}\left[\mathrm{GeV} \mathrm{cm}{ }^{-3}\right]$ & 0.57 & 0.5 \\
\hline$r_{0}[\mathrm{kpc}]$ & 8.3 & 8.3 \\
\hline$\alpha$ & 2 & 2 \\
\hline$\beta$ & 2 & 2 \\
\hline$\gamma$ & 0 & 0 \\
\hline$a[\mathrm{kpc}]$ & 5 & 5 \\
\hline$\varepsilon_{x y}$ & 0.7 & 0.8 \\
\hline$\varepsilon_{z}$ & 0.6 & 0.75 \\
\hline$\phi_{g c}\left[{ }^{\circ}\right]$ & 0 & 90 \\
\hline$M_{200}\left[M_{\odot}\right]$ & $2.8 \times 10^{12}$ & $3.4 \times 10^{12}$ \\
\hline$R_{200}[\mathrm{kpc}]$ & 290 & 310 \\
\hline$\rho_{1}\left[\mathrm{GeV} \mathrm{cm}^{-3}\right]$ & - & 4.5 \\
\hline$R_{1}[\mathrm{kpc}]$ & - & 4.15 \\
\hline$\sigma_{r, 1}[\mathrm{kpc}]$ & - & 4.15 \\
\hline$\sigma_{z, 1}[\mathrm{kpc}]$ & - & 0.17 \\
\hline$\varepsilon_{x y, 1}$ & - & 0.8 \\
\hline$\phi_{1}\left[{ }^{\circ}\right]$ & - & -70 \\
\hline$M_{1}\left[M_{\odot}\right]$ & - & $9.3 \times 10^{9}$ \\
\hline$\rho_{2}\left[\mathrm{GeV} \mathrm{\textrm {cm } ^ { - 3 } ]}\right.$ & - & 1.85 \\
\hline$R_{2}[\mathrm{kpc}]$ & - & 12.9 \\
\hline$\sigma_{r, 2}[\mathrm{kpc}]$ & - & 3.3 \\
\hline$d_{n}[\mathrm{kpc}]$ & - & 4 \\
\hline$\sigma_{z, 2}[\mathrm{kpc}]$ & - & 1.7 \\
\hline$\varepsilon_{x y, 2}$ & - & 0.95 \\
\hline$\phi_{2}\left[{ }^{\circ}\right]$ & - & -20 \\
\hline$M_{2}\left[M_{\odot}\right]$ & - & $\times 10^{10}$ \\
\hline$\chi^{2} /$ d.o.f. & $1206 / 157$ & $144.2 / 157$ \\
\hline probability & 0 & 0.74 \\
\hline
\end{tabular}

cannot be considered an extension of the disk. A viable alternative is the infall of a satellite galaxy (Yanni et al. 2003; Helmi et al. 2003; Rocha-Pinto et al. 2003; Penarrubia et al. 2004; Martinez-Delgado et al. 2005), for which one expects in addition to the visible stars a DM component. From the size of the ring and its peak density one can estimate the amount of DM in the outer ring to be around $9 \times 10^{10}$ solar masses. Since the gamma ray excess is best fitted with a full $360^{\circ}$ of the sky, one can extrapolate the observed $100^{\circ}$ of visible stars to obtain a total visible mass of $\approx 10^{8}-10^{9}$ solar masses (Yanni et al. 2003; Ibata et al. 2003), so the baryonic matter in the outer ring is only a small fraction of its total mass.

The inner ring at $4.2 \mathrm{kpc}$ with a width of $4.2 \mathrm{kpc}$ in radius and $0.2 \mathrm{kpc}$ in $z$ is more difficult to interpret, since the density of the inner region is modified by adiabatic compression (Wilson \& Kalnajs 2001; Blumenthal 1985; Dutton et al. 2003) and interactions between the bar and the halo (Holley-Bockelmann et al. 2003; Athanassoula 2003). However, it is interesting to note that its radius coincide with the ring of cold dense molecular hydrogen gas, which reaches a maximum density at $4.5 \mathrm{kpc}$ and has a width around $2 \mathrm{kpc}$ Gordon \& Burton (1976); Hunter et al. (1997). At the same radius a toroidal structure of dust has been observed, which provides shelter against dissociating UV radiation and allows atomic hydrogen to coalesce at the surfaces into molecular hydrogen. Therefore a ring of molecular hydrogen suggests a gravitational potential well in agreement with the EGRET excess in this region.

\subsection{Comparison with rotation curve}

It is interesting to note that the present analysis is able to trace the mass distribution of DM in our Galaxy, since the mass is given by the WIMP number density $n_{\chi}$ times the WIMP mass $m_{\chi}$. The relative contributions of the rings and pseudo-isothermal profile are obtained from the intensity of the EGRET excess and the absolute normalization of the total mass is obtained by requiring that the local rotation velocity of our solar system at $8.3 \mathrm{kpc}$ is $220 \mathrm{~km} \mathrm{~s}^{-1}$. For the WIMP mass of $60 \mathrm{GeV}$ from the spectrum and the halo parameters from Table 2 we can immediately calculate the mass in the rings and the mass in the pseudo-isothermal profile. For the mass in the outer (inner) ring one finds a value around $9 \times 10^{10}\left(9 \times 10^{9}\right)$ solar masses, which is only a small fraction of the total mass of $3 \times 10^{12}$ solar masses inside a radius $R_{200}$ of $310 \mathrm{kpc}$. The latter radius represents the volume with an averaged overdensity of 200 times the critical density of the universe. However, the mass in the outer ring is about $50 \%$ of the mass of the Galaxy inside its radius. Therefore one expects a significant influence on the rotation curve, which then should show a minimum below and maximum above this radius, since the rotation speed squared is proportional to the derivative of the potential. Note that the absolute value of the masses in the rings is not sensitive to the background model, since the absolute mass scale is set by the normalization to the rotation curve, so a different background model, like the shape from the optimized model will change the boost factor, but not the absolute masses.

A minimum inside the outer ring radius and maximum outside is indeed observed, as shown in Fig. 14. The data were taken from Honma \& Sofue (1997), Brand \& Blitz (1993), Fich et al. (1989), Blitz et al. (1987), Schneider \& Terzian (1983). The contributions from each of the mass terms have been shown separately. The baryonic matter distribution was taken from Olling \& Merrifield (2001). The basic explanation for the negative contribution from the outer ring is that a tracer star at the inside of the ring at $14 \mathrm{kpc}$ feels an outward force from the ring, thus a negative contribution to the rotation velocity. If one just takes the contributions from the visible matter and the isothermal profile without rings, the data cannot be described, as shown on the left hand side of Fig. 15. Here the data points were obtained from the ones in Fig. 14 by taking a weighted average. With the rings a perfect description is obtained, as shown on the right hand side of Fig. 15. Here two rotation curves were calculated: one along the long axis of the 

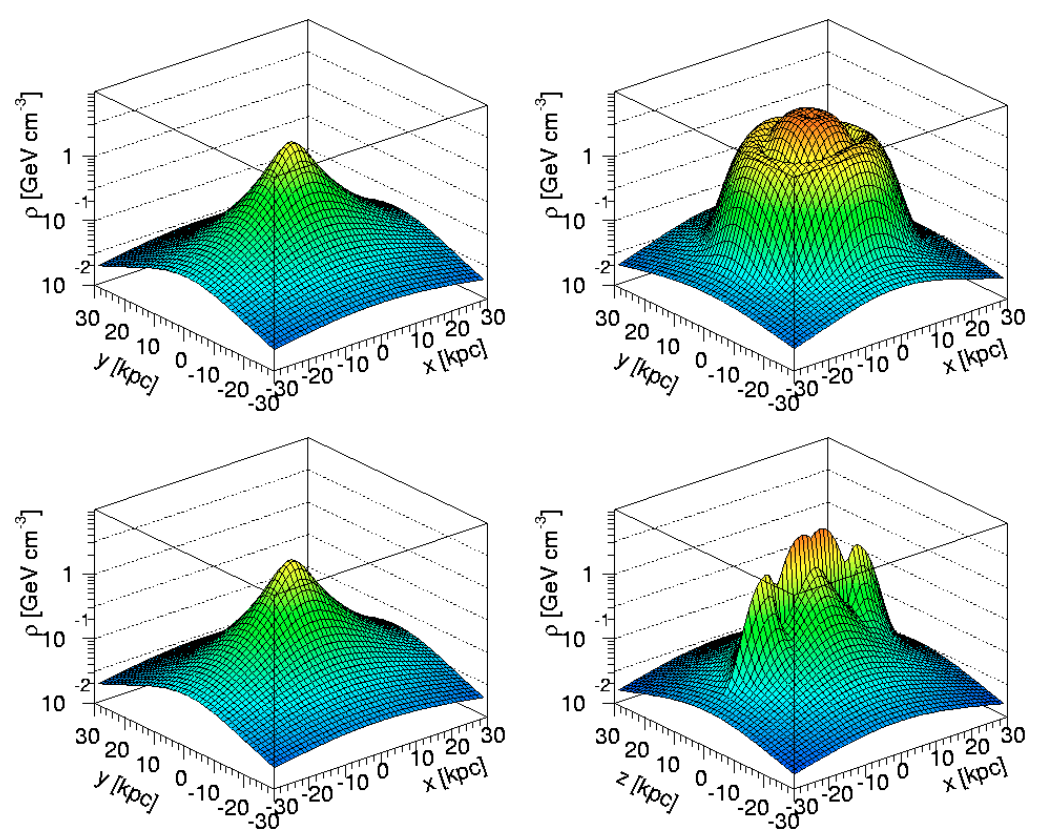

Fig. 12. 3D-presentations of the isothermal haloprofile in the Galactic plane ( $x y$-projection) (top row) and perpendicular to the disk ( $x z$-plane) (bottom row) without (left) and with (right) toroidal ringlike structures at 4 and $14 \mathrm{kpc}$.

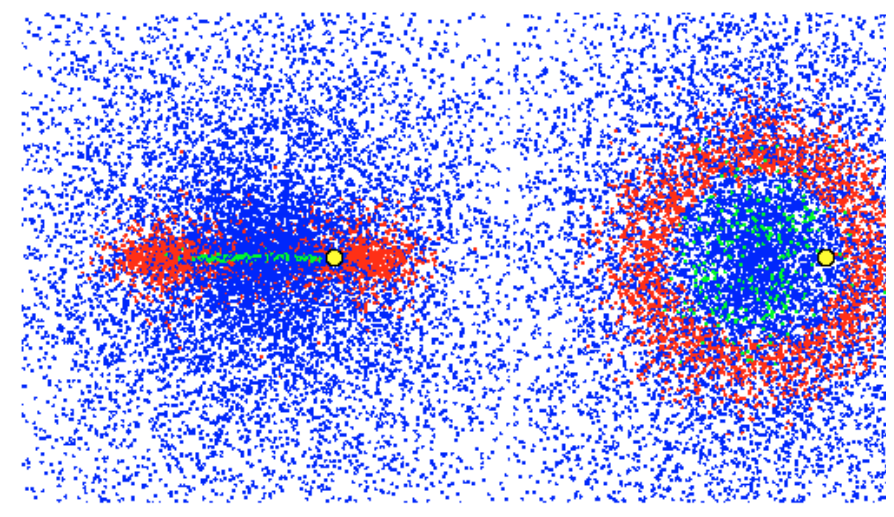

Fig. 13. Visualization of the DM (top) and baryonic matter (bottom) distribution of the Milky Way in an edge-on (left) and top (right) view of the disk. The blue dots mark the modified isothermal profile; the red ones mark the outer and the green ones the inner ring; cyan marks the exponential disk and pink the stellar bulge; the large yellow circle marks the position of the sun; the density of points of each component is proportional to their mass fraction.

triaxial halo profile, which was found to be in the Galactic plane and one perpendicular to this axis in the plane. Since the ellipticity is small ( $\epsilon_{x y} \approx \epsilon_{z} \approx 0.8$, see Table 2$)$, the difference is small.

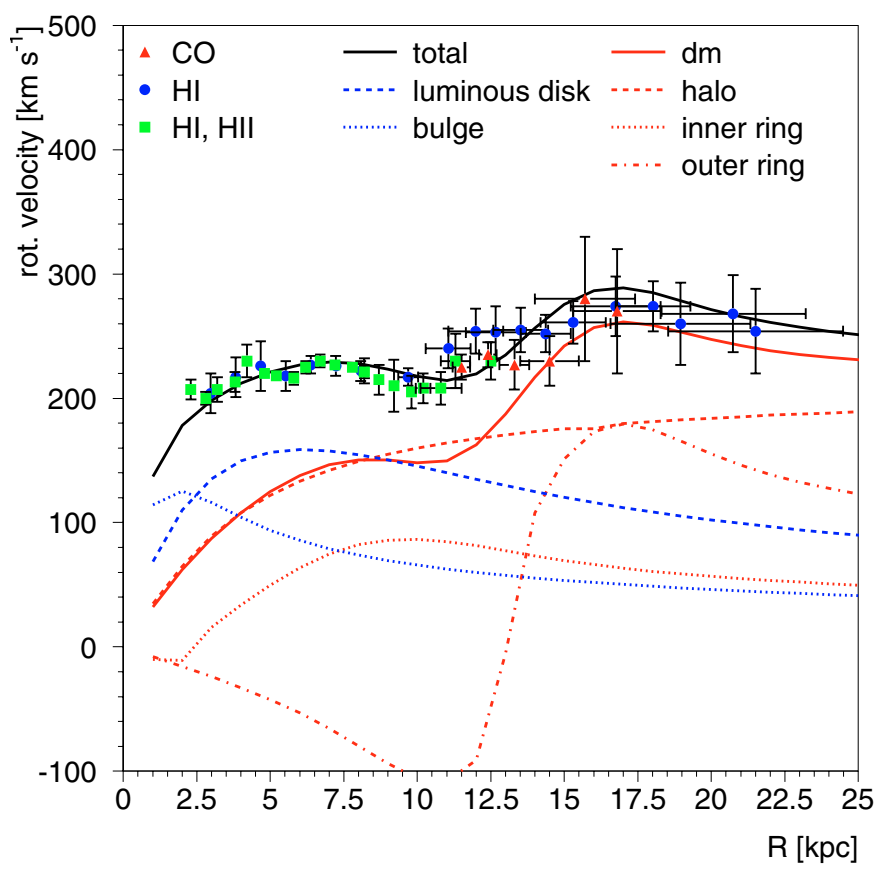

Fig. 14. The rotation curve of the Galaxy for the DM halo profile of Fig. 12. The data are from Honma \& Sofue (1997); Brand \& Blitz (1993); Fich et al. (1989); Blitz et al. (1987); Schneider \& Terzian (1983). The contributions from the individual mass components have been indicated: the upper solid line indicates the contributions from the visible (disk + bulge) plus the DM components. The lower solid line indicates the sum of the DM components (pseudo-isothermal halo, inner and outer ring). Note the negative contribution of the massive ring of DM at $14 \mathrm{kpc}$, which exerts an outward and hence negative force on a tracer inside that ring.

Usually the rotation curves with inhomogeneous mass distributions are calculated by solving the Poisson equation, which 

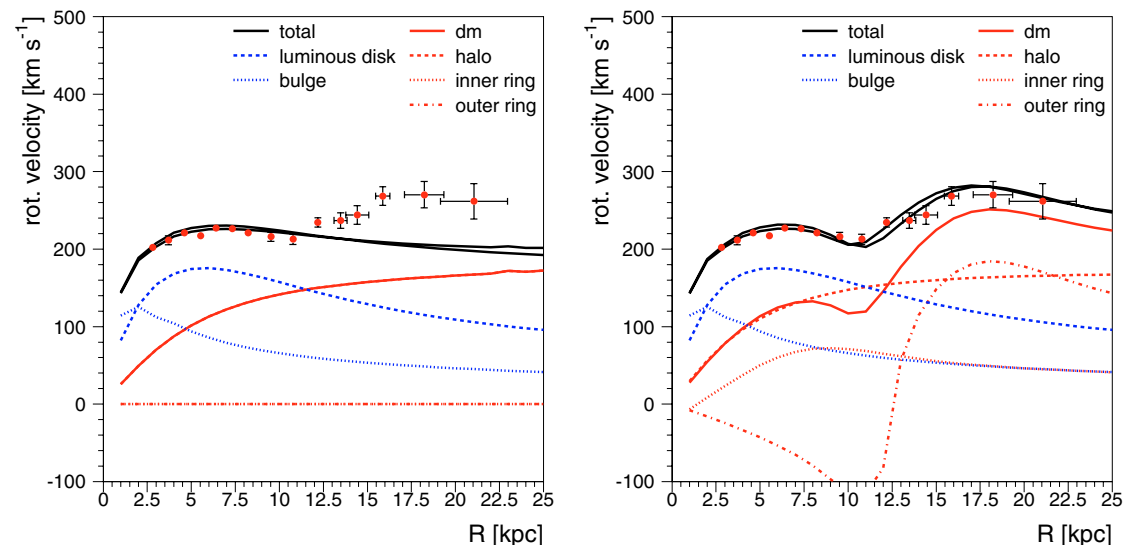

Fig. 15. Rotation curve for the pseudo-isothermal profile without (left) and with (right) rings in the Galactic plane; here the different data sets on rotation velocities and nomenclature are the same as in Fig. 14, but for clarity a weighted average has been taken. One can see that the pseudo-isothermal profile without rings does not provide a good fit to the data, while the inclusion of the toroidal substructures provides a perfect fit. The rotation curves (solid black lines) are calculated in two perpendicular directions: along the long and medium axis of the triaxial halo, which both lie in the plane of the disk.

yields the gravitational potential at a given position: $\Phi(r, \theta, \phi)$. The rotation velocity for a circular orbit at a radius $r$ can then be calculated by requiring that the resulting gravitational force on a tracer star equals the centrifugal force, i.e.

$v^{2} / r=F_{G} / m \equiv \mathrm{d} \Phi(r) / \mathrm{d} r$.

However, the contribution from ringlike structures is not easily obtained from a standard Poisson solver, since these are usually optimized for spherical symmetries. They did not converge for the ringlike structures in our case. Therefore the derivative of the potential instead of the potential was calculated numerically, which is what is needed for the rotation curve and avoids an additional numerical derivation.

To be more precise, the following was done. The gravitational potential $\Phi$ of the Poisson equation can be written in spherical coordinates $(x=r \cos \phi \sin \theta, y=r \sin \phi \sin \theta, z=$ $r \cos \theta$ ) as:

$$
\begin{aligned}
\Phi(r, \theta, \phi)= & -\int_{0}^{\infty} r^{\prime 2} \mathrm{~d} r^{\prime} \int_{-1}^{1} \mathrm{~d} \cos \theta^{\prime} \\
& \times \int_{0}^{2 \pi} \mathrm{d} \phi^{\prime} \rho\left(r^{\prime}, \theta^{\prime}, \phi^{\prime}\right)\left(r^{2}+r^{\prime 2}-2 r r^{\prime} \sin \theta \sin \theta^{\prime}\right. \\
& \left.\times \cos \left(\phi-\phi^{\prime}\right)-2 r r^{\prime} \cos \theta \cos \theta^{\prime}\right)^{-1 / 2}
\end{aligned}
$$

or in the plane in the direction $\phi=0, \theta=\pi / 2$ :

$$
\begin{aligned}
\Phi(r, \pi / 2,0)= & -\int_{0}^{\infty} r^{\prime 2} \mathrm{~d} r^{\prime} \int_{-1}^{1} \mathrm{~d} \cos \theta^{\prime} \\
& \times \int_{0}^{2 \pi} \mathrm{d} \phi^{\prime} \frac{\rho\left(r^{\prime}, \theta^{\prime}, \phi^{\prime}\right)}{\sqrt{r^{2}+r^{\prime 2}-2 r r^{\prime} \sin \theta^{\prime} \cos \left(\phi-\phi^{\prime}\right)}}
\end{aligned}
$$

Note that $\rho$ includes all masses. The rotation velocity for a circular orbit at a radius $r$ can then be calculated in the direction $\phi=0, \theta=\pi / 2$ (using Eq. (7)):

$$
\begin{aligned}
\frac{v^{2}(r)}{r}= & \frac{\mathrm{d} \Phi(r)}{\mathrm{d} r}=\int_{0}^{\infty} r^{\prime 2} \mathrm{~d} r^{\prime} \int_{-1}^{1} \mathrm{~d} \cos \theta^{\prime} \\
& \times \int_{0}^{2 \pi} \mathrm{d} \phi^{\prime} \frac{\rho\left(r^{\prime}, \theta^{\prime}, \phi^{\prime}\right)\left(r-r^{\prime} \sin \theta^{\prime} \cos \left(\phi-\phi^{\prime}\right)\right)}{\left(r^{2}+r^{\prime 2}-2 r r^{\prime} \sin \theta^{\prime} \cos \left(\phi-\phi^{\prime}\right)\right)^{3 / 2}} .
\end{aligned}
$$

This threefold integral was integrated numerically to obtain the contribution from all mass elements in the halo. The contributions of the bulge, disk and DM contributions from the isothermal halo plus rings have been indicated separately in Fig. 14. The negative contribution from a ring is expressed by the fact that the derivative of the gravitational potential $\Phi$ changes its sign, when crossing the maximum of the ring and so does the contribution to $v^{2}$ (see term $r-r^{\prime}$ in numerator of Eq. (10)). This implies an outward gravitational force exerted by the ring for a tracer inside the ring and an inward force for a tracer outside the ring. The hitherto mysterious change of sign of the slope near $r=1.3 r_{0}$ finds then its natural explanation in the large ring of DM at $r=14 \mathrm{kpc}$, whose mass is determined by the excess of energetic gamma rays.

\subsection{Galactic parameters}

From the baryonic density, as derived from the rotation curve and the DM profile determined in this paper, one can determine the following basic properties of our Galaxy:

- The radius containing an average density 200 times the critical density equals $R_{200}=310 \mathrm{kpc}$.

- The total DM mass inside this radius is $M_{200}=3.0 \times$ $10^{12} M_{\odot}$ to be compared with a visible mass of $5.5 \times$ $10^{10} M_{\odot}$.

- The inner (outer) ring contribute 0.3 (3) \% to the total DM mass.

- The fraction $f_{\mathrm{d}}=M_{\text {baryonic }} / M_{200}=0.02$.

- The concentration parameter $c=R_{200} / a=310 / 5=63$.

It should be remembered that these parameters were obtained assuming an isothermal profile for the DM with a constant boost factor. Assuming a smaller boost factor in the bulge because of tidal disruptions there, will increase the mass in the center, since the flux is proportional to $B(r)\left\langle\rho^{n}\right\rangle$, as discussed before. This reduces the DM mass in the outer regions, so the numbers given above should be considered an upper limit, but these parameters are well inside the range found for 
other galaxies with an isothermal profile (Jimenez et al. 2003) and earlier mass estimates of the Galaxy (Wilkinson \& Evans 1999).

\section{Possible objections to the DMA interpretation}

The DMA interpretation of the EGRET excess would mean that DM is not so dark anymore, but DM is visible from the 30-40 flashes of energetic gamma rays for each annihilation. This would be great, but are there more mundane explanations? Attempts to modify the electron and proton spectra from the locally measured spectra do not describe the shape of the EGRET data in all sky directions, as discussed in detail before by comparing the EGRET data with the "optimized model". Here we summarize some other possible objections.

1. Are the EGRET data reliable enough to make such strong conclusions? The EGRET detector was calibrated in a quasi mono-energetic gamma ray beam at SLAC, so its response is well known (Thompson et al. 1987). Also the monitoring during the flight was done carefully (Esposito et al. 1999). We have only used data in the energy range between 0.07 and $10 \mathrm{GeV}$, where the efficiency is more or less flat. So the 9 years flight provided accurate and reliable data, especially it would be hard to believe in an undetected calibration problem, which would only effect the data above $0.5 \mathrm{GeV}$ and fake the gamma ray spectrum from the fragmentation of mono-energetic quarks.

2. The gamma ray spectrum above $0.07 \mathrm{GeV}$ starts to be dominated by pp-interactions and is therefore strongly dependent on the proton energy spectrum. This cosmic ray spectrum was measured only locally in the solar neighborhood. Could a harder spectrum near the Galactic center, where protons can be accelerated by the many supernovae there, explain the EGRET excess? No, first of all the diffusion times are much shorter than the energy loss times of protons with energies above a few $\mathrm{GeV}$, so one expects everywhere the same energy spectrum. This is proven by the fact, that the gamma ray spectrum for the Galactic center and the Galactic anti-center can be described by the same background shape.

3. Is the background well enough known to provide evidence for DMA? The background is dominated by pp-collisions with a reasonably well known shape and fitting the normalization yields a "self-calibrating" background. Trying to obtain a harder gamma ray spectrum by hardening the proton spectrum increases usually not only the high energy gamma rays, but also contributes to the low energy part of the spectrum. Fitting this "wrong" shape with a free normalization reduces then the low energy excess again and recovers the high energy excess. Note that this "self-calibration" of the background also takes care of gas clouds, ringlike or asymmetric structures in the background, uncertainties in the absolute value of the total cross sections, etc.

An alternative way of formulating the problem of models without DMA: if the shape of the EGRET excess can be explained perfectly in all sky directions by a gamma contribution originating from the fragmentation of monoenergetic quarks, it is very difficult to replace such a contribution by an excess from nuclei (quarks) or electrons with a steeply falling energy spectrum, especially if one takes into account that the spatial distribution of gamma rays from DMA is different from the gamma rays from the background.

4. Is it possible to explain the excess in diffuse gamma rays with unresolved point sources? This is unlikely, first of all since the known point sources (Hartmann et al. 1999) are only a small fraction of the diffuse gamma rays and the majority of the resolved sources has a rather soft spectrum, typically well below $1 \mathrm{GeV}$, as can be seen from the plots in the Appendix. If this part of the spectrum would be dominated by unresolved sources, then the diffuse component below $1 \mathrm{GeV}$ would become lower after subtracting the hypothetical unresolved sources, which in turn would lead to a lower normalization of the background and a correspondingly stronger excess for a fixed background shape. So arguing against DMA by unresolved sources goes in the wrong direction.

5. Is one not over-interpreting the EGRET data by fitting so many parameters for the different components: triaxial halo, inner ring and outer ring? No, first of all the excess and enhancement in a ringlike structure at $14 \mathrm{kpc}$ was already discovered in the original paper by Hunter et al. (1997). What we did is just trying to see if the excess fits: a) a single WIMP mass in all directions; b) an isothermal DM profile plus the substructure; c) the Galactic rotation curve. The DM halo components are determined by independent sky directions: the outer ring parameters are determined mainly by 30 sky directions towards the Galactic anti-center, the inner ring parameters by ca. 15 sky directions towards the Galactic center and the triaxial halo parameters by ca. 130 sky directions out of the Galactic plane. And the most remarkable thing is that all these independent sky directions all show an excess, which can be explained by a single WIMP mass around $60 \mathrm{GeV}$. This is like having 180 independent experiments at an accelerator all saying we see a significant excess of gamma rays corresponding to $\pi^{0}$ production from mono-energetic quarks. Then asked what mass they need to describe the excess, they all say $60 \mathrm{GeV}$ !

6. The outer rotation curve of our Galaxy has large uncertainties from the distance $r_{0}$ between the Sun and the Galactic center and is determined with a different method than the inner rotation curve. Can this fake the good agreement between the calculated rotation curve from the gamma ray excess and the measured rotation curve? The outer rotation curve indeed depends strongly on $r_{0}$, as shown by Honma $\&$ Sofue (1997), who varied $r_{0}$ between 7 and $8.5 \mathrm{kpc}$. At present one knows from the kinematics of the stars near the black hole at the center of our Galaxy that $r_{0}=8 \pm 0.4 \mathrm{kpc}$ (Eisenhauer et al. 2003), so the distance is already reasonably well known. But whatever the value of $r_{0}$, the change in slope around $1.3 r_{0}$ is always present, indicating a ringlike DM structure is always needed. Furthermore the outer rotation curve shows first the same decrease as the inner 
rotation curve and only then changes the slope, so the different methods agree between $r_{0}$ and $1.3 r_{0}$

7. The outer ring at $14 \mathrm{kpc}$ has a mass around $9 \times 10^{10}$ solar masses. This is around $50 \%$ of the total mass inside the ring and one may worry about the disk stability of the Milky Way by the infall of such a heavy Galaxy. However, large spiral galaxies show bumps of similar size (Sofue 2000), so it seems not to be uncommon to have masses of this size forming ringlike structures.

8. One observes a ring of molecular hydrogen near the inner ring and a ring of atomic hydrogen near the outer ring. Could this excess of hydrogen not be responsible for the excess of the gamma rays? No, our method of fitting only the shapes with a free normalization implies that this analysis is insensitive to density fluctuations of the background, which change the normalization, not the shape.

9. How can one be sure that the outer ring originated from the tidal disruption of a rather massive satellite galaxy, so one can expect an enhanced DM density in the ring? One finds three independent ringlike structures: stars, atomic hydrogen gas and excess of gamma radiation. The stars show a scale height of several $\mathrm{kpc}$ and a low velocity dispersion, so they cannot be part of the Galactic disk. Therefore the infall of a satellite galaxy is the natural explanation. Since the tidal forces are proportional to $1 / r^{3}$, the satellite will be disrupted most strongly at its pericenter, which can lead to DM density enhancements at the pericenter after a few orbits (Hayashi et al. 2003). Some of the stars and gas may be caught in this potential well. All three are found at $14 \mathrm{kpc}$ with the stars all being old and more than $90 \%$ of the mass being DM as deduced from the strong EGRET excess at this radius.

10. Is it not peculiar that if a ringlike structure originates from the infall of satellite galaxy, that it lies in the plane of the Galaxy? No, in principle the infall can happen in all directions with respect to the plane, but the angular momenta of the inner halo and a baryonic disk tend to align after a certain time by tidal torques (Bailin et al. 2005).

\section{Summary}

If Dark Matter is a thermal relic from the early Universe, then it is known to annihilate, since the small amount of relic density measured nowadays requires a large reduction in its number density. The annihilation cross section can be obtained directly from its inverse proportionality to the relic density, the latter being well known from precision cosmology experiments (Spergel et al. 2003). The annihilation into quark pairs will produce $\pi^{0}$ mesons during the fragmentation into hadrons, which in turn will decay into gamma rays. Since $\mathrm{DM}$ is cold, i.e. non-relativistic, the fragmenting quarks have an initial energy equal to the WIMP mass. The gamma spectrum from such mono-energetic quarks is well known from electron-positron colliders, which produce exactly such states. For heavy WIMP masses the gamma spectrum is considerably harder than the background spectrum, mainly from $\pi^{0}$ mesons produced in pp-collisions from cosmic rays with gas in the disk. Such an excess of hard gamma rays has indeed been observed by the EGRET satellite and the relative contributions from background and DM annihilation signal can be obtained by fitting their different shapes with a free normalization factor for background and signal.

The results of the analysis can be summarized as follows:

- By analyzing the EGRET data in 180 independent sky directions we find first of all an excess in each direction, as expected for DMA, and secondly, the spectral shape of the excess is the same in each direction and corresponds to a WIMP mass around $60 \mathrm{GeV}$.

- For each direction 8 energy points between 0.07 and $10 \mathrm{GeV}$ have been used, so more than 1400 independent data points are available, which are more than enough to reconstruct the DM halo profile from the flux distribution of the excess in the 180 sky directions. The profile can be well fitted with an almost spherical pseudo-isothermal profile with a core radius of $5 \mathrm{kpc}$ and substructure in the disk in the form of two toroidal structures at radii of 4 and $14 \mathrm{kpc}$. The excess at $r=14 \mathrm{kpc}$ is correlated with the "ghostly" ring of stars, thought to originate from the tidal disruption of a satellite galaxy. Since such a satellite galaxy can carry a large amount of DM, the excess of the diffuse gamma rays in this region is naturally explained by DMA. The inner structure at a radius of $4.2 \mathrm{kpc}$ coincides with the ring of molecular hydrogen gas, and dust. Molecular hydrogen forms preferentially in the presence of dust, which provides shelter against dissociating UV radiation and enhances the formation rate. The gathering of dust in this region can be explained by the potential well from DM. Note that the structures in the halo profile are completely uncorrelated: the outer ring is mainly determined by the flux of the excess in the disk at large longitudes, i.e. opposite to the Galactic center, while the inner ring is determined from the longitudes below $40^{\circ}$, i.e. towards the inner Galaxy. The isothermal profile is mainly determined from the fluxes out of the plane, i.e. at latitudes above $10^{\circ}$.

- The flux of the excess determines the halo profile, i.e. the number density $n_{\chi}$ of the WIMPs. Together with the WIMP mass $m_{\chi}$ from the spectrum of the excess one can reconstruct the DM mass distribution $\left(=n_{\chi} \cdot m_{\chi}\right)$ in the Galaxy, which in turn can be used - in combination with the visible matter - to calculate the rotation curve. The result explains indeed the hitherto mysterious change of slope in the outer rotation curve.

- The nearby toroidal structures of DM in the disk result in an enhanced DMA in the disk, which is orthogonal to the prejudice that DM should be distributed more or less spherically. However, N-body simulations indicate that the angular momenta of the inner DM halo and disk get aligned (Bailin et al. 2005), so ringlike structures from the tidal disruption of satellite galaxies will end up in the plane of the disk, thus explaining naturally the minima and maxima observed in the outer rotation curves of many galaxies.

The results mentioned above make no assumption on the nature of the Dark Matter, except that its annihilation produces hard gamma rays from quark fragmentation. The fitted 
normalization of the background flux comes out to be close to the absolute prediction of the GALPROP conventional propagation model of our Galaxy (Strong et al. 2000), while the normalization of the DM signal corresponds to a boost factor from 20 upwards. Such a boost factor from the clustering of DM was calculated with respect to the annihilation cross section from Eq. (1), which is the cross section at the freeze-out temperature of a few $\mathrm{GeV}$. At the present time the temperature of the universe is much lower, which could reduce the annihilation cross section, thus increasing the boost factor.

A good WIMP candidate is the neutralino of Supersymmetry. For the WIMP mass in the range of $50-100 \mathrm{GeV}$ this has very much the properties of a spin $1 / 2$ photon, which would imply that DM is the supersymmetric partner of the cosmic microwave background (CMB). The present analysis is perfectly consistent with such a scenario, if the supersymmetric partners of the quarks and leptons are around $1 \mathrm{TeV}$. Details about the connection with Supersymmetry have been discussed elsewhwere (De Boer 2005; De Boer 2005a,b; Sander 2005).

It should be emphasized that the excess of diffuse gamma rays has a statistical significance of at least $10 \sigma$ if compared with the conventional shape of the background. This combined with all features mentioned above provides an intriguing hint that this excess is a) indeed indirect evidence for Dark Matter Annihilation and b) traces the DM in our Galaxy, as proven by the fact that we can reconstruct the rotation curve of our Galaxy from the gamma rays.

Acknowledgements. We thank I.V. Moskalenko, O. Reimer and A. Strong for sharing with us all their knowledge about our Galaxy and the EGRET data and allowing us to use their implementation of the EGRET analysis software in the GALPROP program.

We also like to thank the EGRET Science Team for their hard work for collecting and calibrating the data and the NASA for their support in making satellite data publicly available.

This work was supported by the BMBF (Bundesministerium für Bildung und Forschung) via the DLR (Deutsches Zentrum für Luft- und Raumfahrt), a grant from the DFG (Deutsche Forschungsgemeinschaft, Grant 436 RUS 113/626/0-1), RFBR (Russian Foundation for Basic Research, Grant 05-02-17603), and the Heisenberg-Landau Program.

\section{References}

Aloisio, R., Blasi, P., \& Olinto, A. V. 2004, JCAP, 0405, 007 [arXiv: astro-ph/0402588]

Athanassoula, E. 2003, in IAUS, ed. S. D. Ryder, D. J. Pisano, M. A. Walker, \& K. C. Freeman, 220, 255 [arXiv: astro-ph/0312155]

Bailin, J., Kawata, D., Gibson, B. K., et al. 2005, ApJ, 627, L17

Berezinsky, V., Gurevich, A. V., \& Zybin, K. P. 1992, Phys. Lett. B294, 221

Berezinsky, V., Berezinsky, V., Bottino, A., \& Mignola, G. 1994, Phys. Lett., B325, 136

Berezinsky, V., Dokuchaev, V., \& Eroshenko, Y. 2004

[arXiv: astro-ph/0412305]

Bergström, L. 2000, Rep. Prog. Phys., 63, 793

Bergström, L., Ullio, P., \& Buckley, J. H. 1998, Astropart. Phys., 9, 137

Bergström, L., Edsjö, J., Gondolo, P., \& Ullio, P. 1999, Phys. Rev., D59, 043506
Bergström, L., Edsjö, J., \& Ullio, P. 2001, Phys. Rev. Lett., 87, 251301

Bertone, G., Sigl, G., \& Silk, J. 2002, MNRAS, 337, 98

Bertone, G., Hooper, D., \& Silk, J. 2004, Phys. Rept. 2005, 405, 279

Blitz, L., Fich, M., \& Stark, A. A. 1987, in Proc. IAU Symp., ed. B. H. Andrew, 87, 213

Blumenthal, G. R., Faber, S. M., Flores, R., \& Primack, J. R. 1985, ApJ, 301, 27

Brand, J., \& Blitz, L. 1993, A\&A, 275, 67

Cesarini, A., Fucito, F., Lionetto, A., Morselli, A., \& Ullio, P. 2004 Astropart. Phys., 21, 267

Crane, J. D., Majewski, S. R., Rocha-Pinto, H. J., et al. 2003, ApJ, 594, L119

de Boer, W., Herold, M., Sander, C., et al. 2004 [arXiv: astro-ph/0408272]

de Boer, W. 2005, New Astron. Rev., 49, 213

de Boer, W. 2005, to be published in the Proc. of the XXXXth Rencontres de Moriond on Electroweak Interactions, La Thuile, March 2005 [arXiv: astro-ph/0506447]

de Boer, W. 2005, W. de Boer, Talk at 2005 International Linear Collider Workshop (LCWS 2005), Stanford, California, 18-22 Mar. 2005 [arXiv:hep-ph/0508108]

Diemand, J., Moore, B., \& Stadel, J. 2005, Nature, 433, 389

Diemand, J., Moore, B., Stadel, J., \& Carollo, M. 2005 [arXiv: astro-ph/0504215]

Dutton, A. A., Courteau, S., Carignan, C., \& de Jong, R. 2003 [arXiv: astro-ph/0310001]

Edsjö, J. 2002, [arXiv: astro-ph/0211354]

Eisenhauer, F., Schödel, R., Genzel, R., et al. 2003, ApJ, 597, L121

Ellis, J., Hagelin, J. S., Nanopoulos, D. V., Olive, K., \& Srednicki, M. 1984, Nucl. Phys. B, 238, 453

Esposito, J. A., Bertsch, D. L., Chen, A. W., et al. 1999, ApJS, 123, 203

Fargion, D., Konoplich, R., Grossi, M., \& Khlopov, M. Y. 2002, Aph, 12,307

Fich, M., Blitz, L., \& Stark, A. A. 1989, ApJ, 342, 342

Gordon, M. A., \& Burton, W. B. 1976, ApJ, 208, 346

Hartman, R. C., et al. 1999, ApJ, S123, 79

Hayashi, E., Navarro, J. F., Taylor, J. E., Stadel, J., \& Quinn, T. 2003, ApJ, 584, 541

Helmi, A., Navarro, J. F., Meza, A., Steinmetz, M., \& Eke, V. R. 2003, ApJ, 592, L25

Holley-Bockelmann, K., Weinberg, M., \& Katz, N. 2003, MNRAS, 363, 991

Honma, M., \& Sofue, Y. 1997, Publ. Astr. Soc. Japan, 49, 453

Hooper, D., \& Dingus, B. 2002 [arXiv: astro-ph/0212509]

Hunter, S. D., et al. 1997, ApJ, 481, 205

Ibata, R. A., Irwin, M. J., Lewis, G. G., Ferguson, A. M. N., \& Tanvir, N. 2003, MNRAS, 340, L21

Jimenez, R., Verde, L., \& Oh, S. P. 2003, MNRAS, 339, 243

Jungman, G., \& Kamionkowski, M. 1995, Phys. Rev. D, 51, 3121

Jungman, G., Kamionkowski, M., \& Griest, K. 1996, Phys. Rep., 267, 195

Kamae, T., Abe, T., \& Koi, T. 2005, ApJ, 620, 244

Kolb, E., \& Turner, M. S. 1990, The Early Universe (Addison Wesley)

Martinez-Delgado, D., Penarrubia, J., Dinescu, D. I., Butler, D. J., \& Rix, H. W. 2005 [arXiv: astro-ph/0506012]

Morselli, A. 2002, Int. J. Mod. Phys. A, 17, 1829

Moskalenko, I. V., Strong, A. W., \& Reimer, O. 1998, A\&A, 338, L75

Navarro, N. F., Frenk, C. S., \& White, S. D. 1996, ApJ, 490, 493

Newberg, H. J., Yanny, B., Rockosi, C., et al. 2002, ApJ, 569, 245

Olling, R. P., \& Merrifield, M. R. 2001, MNRAS, 326, 164

Rocha-Pinto, H. J., Majewski, S. R., Skrutskie, M. F., \& Crane, J. D. 2003 [arXiv: astro-ph/0307258] 
Penarrubia, J., Martinez-Delgado, D., Rix, H. W., et al. 2004 [arXiv: astro-ph/0410448]

Sander, C. 2005, Ph.D. Thesis (University of Karlsruhe)

Schneider, S. E., \& Terzian, Y. 1983, ApJ, L61, 274

Sjöstrand, T., Lönnblad, L., Mrenna, S., \& Skands, P. 2003, PYTHIA 6.3 physics and manual [arXiv:hep-ph/0308153]

Sjöstrand, T., Edén, P., Friberg, C., et al. 2001, Computer Phys. Commun. 135, 238

Sofue, Y. 2000, in Galaxy Disks and Disk Galaxies, ASP Conf. Ser., Vatical Conf. Rome 2000, ed. J. Funes, \& E. M. Corsini [arXiv:astro-ph/0010595]

Spergel, D. N., Verde, L., Peiris, H. V., et al. 2003, ApJS, 148, 175

Sreekumar, P., Bertsch, D. L., Dingus, B. L., et al. (EGRET Collaboration) 1998, ApJ, 494, 523

Strong, A. W., \& Moskalenko, I. V. 1998, ApJ, 509, 212

Strong, A. W., Moskalenko, I. V., \& Reimer, O. 2000, ApJ, 537, 763 (Erratum-ibid. 541, 1109);Details on the latest GALPROP versions can be found at http://www.mpe.mpg.de/ aws/propagate.html
Strong, A. W., Moskalenko, I. V., \& Reimer, O. 2004, ApJ, 613, 962 Sumner, T. J. 2002, www . livingreviews .org/Articles/Volume5/ 2002-4sumner; Living Reviews in Relativity published by the Max Planck Institute for Gravitational Physics, Albert Einstein Institute, Germany

Tasitsiomi, A., Gaskins, J., \& Olinto, A. V. 2004, ApJ, 21, 637

Thompson, D. J., Bertsch, D. L., Fichtel, C. E., Hartman, R. C., \& Favale, A. 1987, IEEE Trans. Nucl. Sci., 34, 36

Thompson, D. J., Bertsch, D. L., \& O’Neal, R. H. 2005, ApJS, 157, 324

Tyler, C. 2002, Phys. Rev. D, 66, 023509

Ullio, P., Bergstrom, L., Edsjo, J., \& Lacey, C. G. 2002, Phys. Rev. D, 66,123502

Wilkinson, M. I., \& Evans, N. W. 1999, MNRAS, 310, 645

Wilson, G., \& Kalnajs, A. 2001, ASP Conf. Ser., in press

Wilson, G. 2003, thesis, Research School of Astronomy and Astrophysics, Australian National University (Canberra)

Yanny, B., et al. 2003, ApJ, 588, 824 (Erratum-ibid. 605, 575) 
W. de Boer et al.: Diffuse galactic gamma rays as tracer of dark matter, Online Material $p 1$

\section{Online Material}


W. de Boer et al.: Diffuse galactic gamma rays as tracer of dark matter, Online Material $p 2$

\section{Appendix A: Fits to the EGRET data for $\mathbf{1 8 0}$ independent sky directions.}

The fits of the shapes of the gamma ray spectrum from the conventional background model and DMA signal to the EGRET data are shown for 180 regions of the sky: the longitude distributions are split into bins of $8^{\circ}$ for four different latitude ranges (absolute values of latitude: $0-5^{\circ}, 5-10^{\circ}, 10-20^{\circ}, 20-90^{\circ}$ ), so one has $4 \times 45=180$ angular bins. The background scalings and the boost factors are free parameters. The contribution from the extragalactic background, as determined by Sander (2005), and the spectra of the subtracted EGRET point sources from Hartmann et al. (1999) are shown as well. 
W. de Boer et al.: Diffuse galactic gamma rays as tracer of dark matter, Online Material p 3

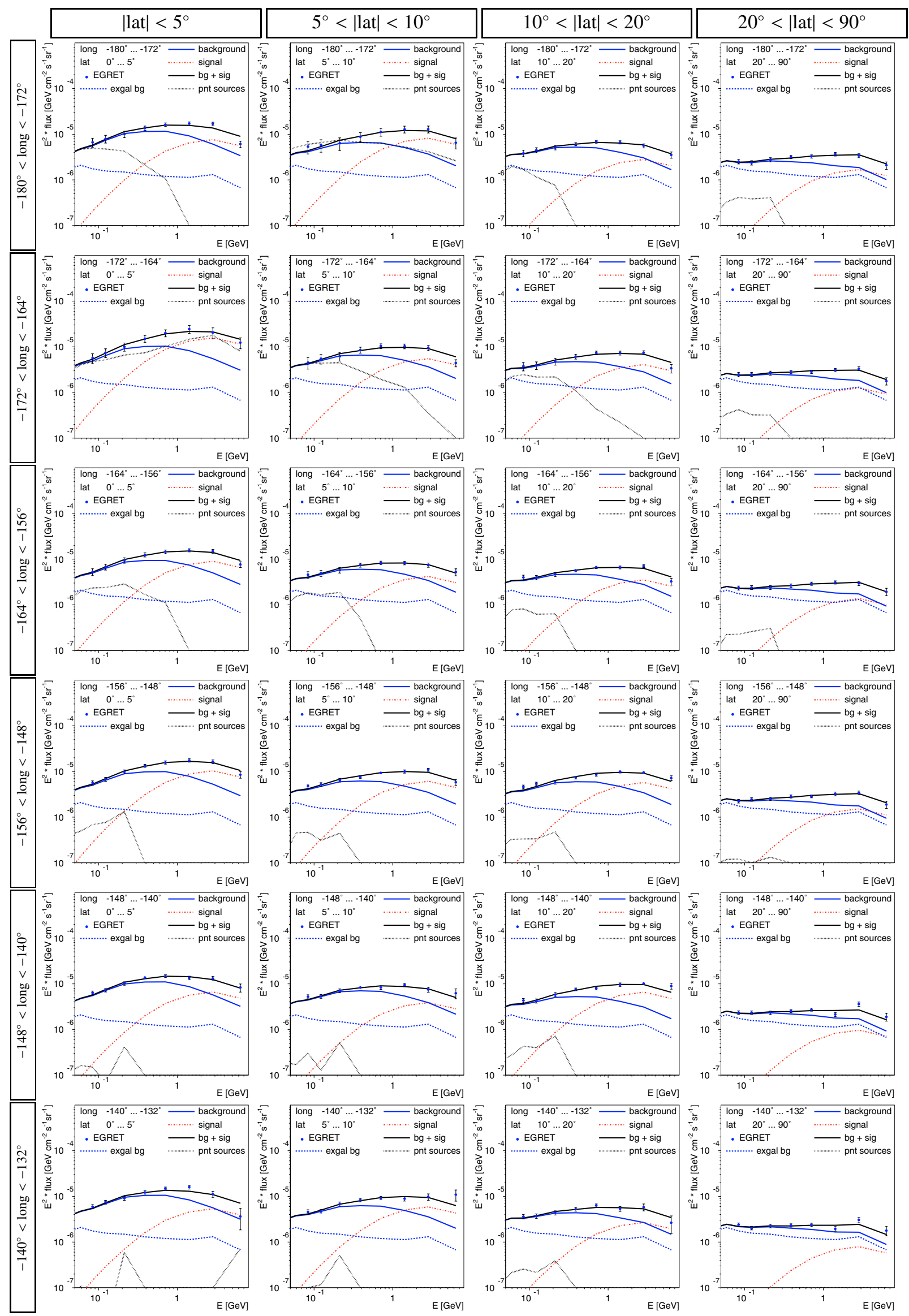


W. de Boer et al.: Diffuse galactic gamma rays as tracer of dark matter, Online Material $p 4$

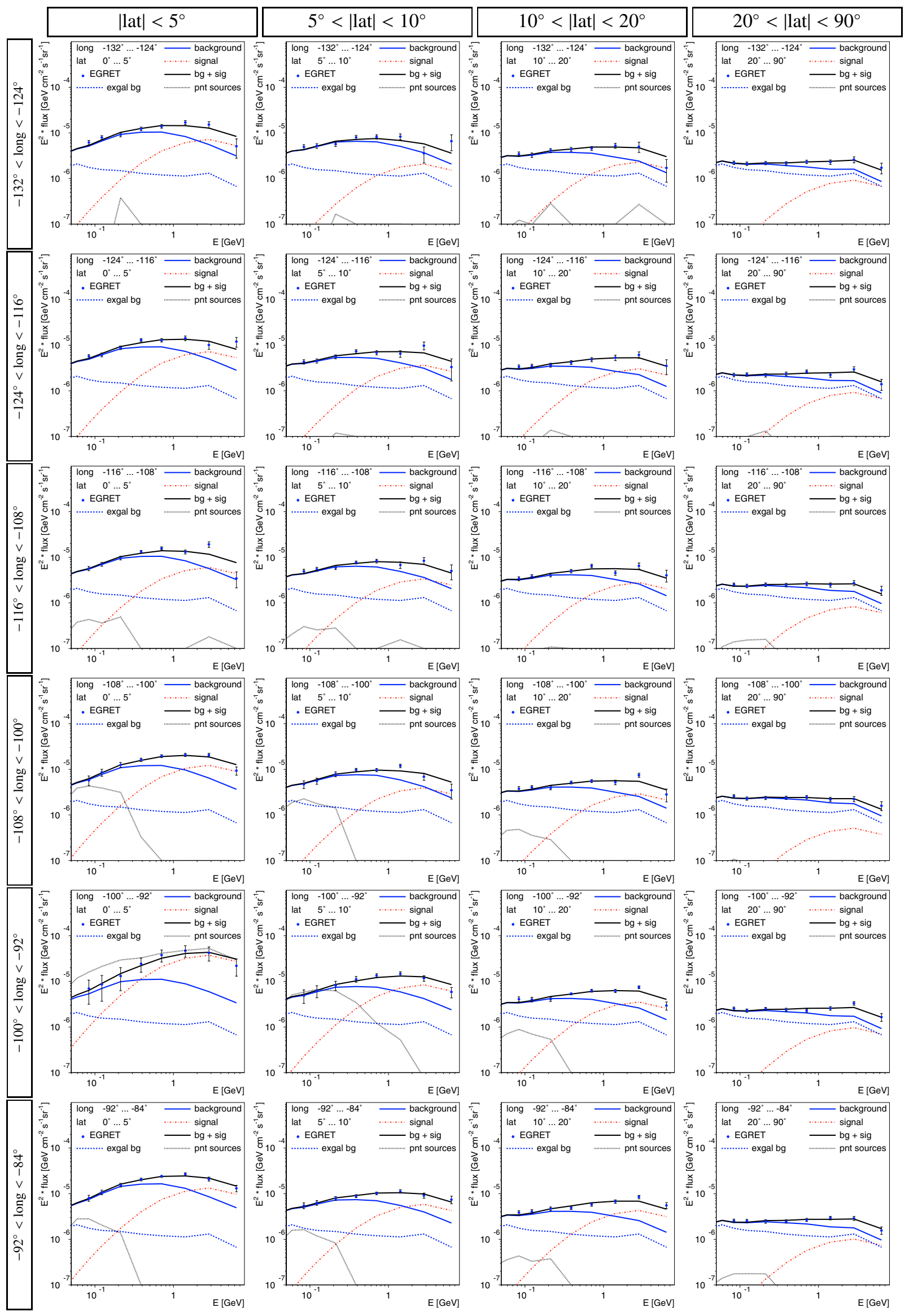


W. de Boer et al.: Diffuse galactic gamma rays as tracer of dark matter, Online Material p 5

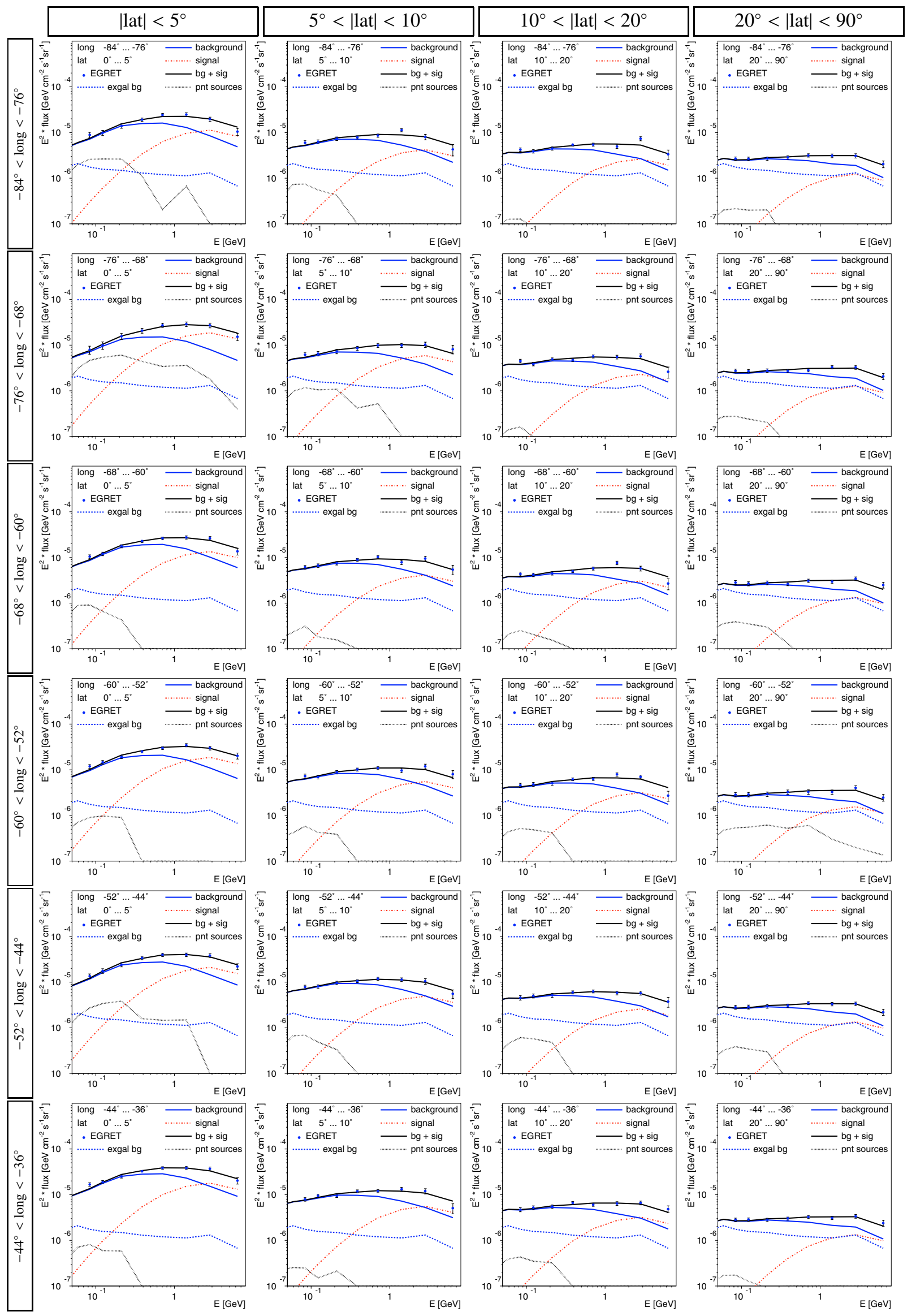


W. de Boer et al.: Diffuse galactic gamma rays as tracer of dark matter, Online Material p 6

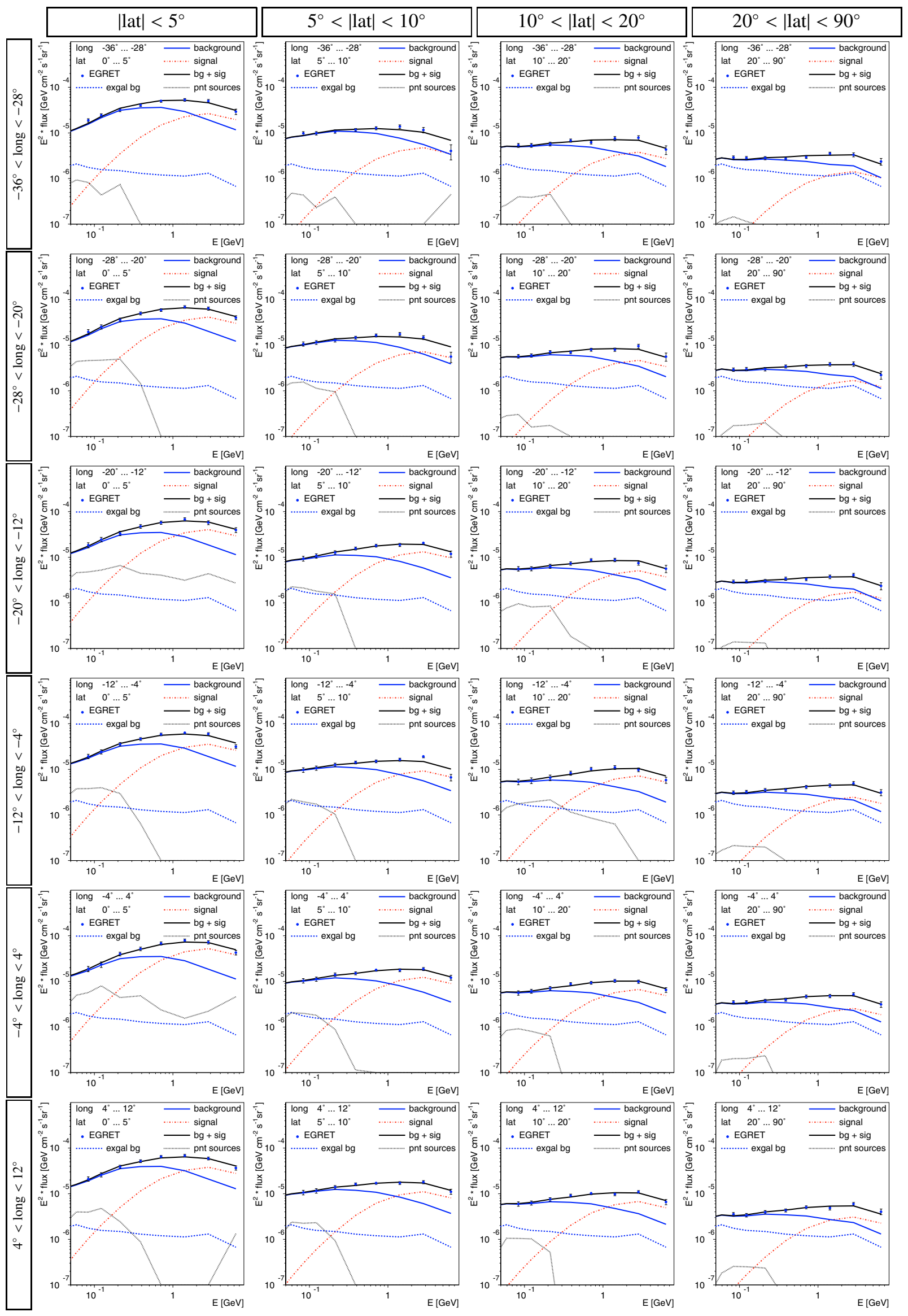


W. de Boer et al.: Diffuse galactic gamma rays as tracer of dark matter, Online Material $p 7$

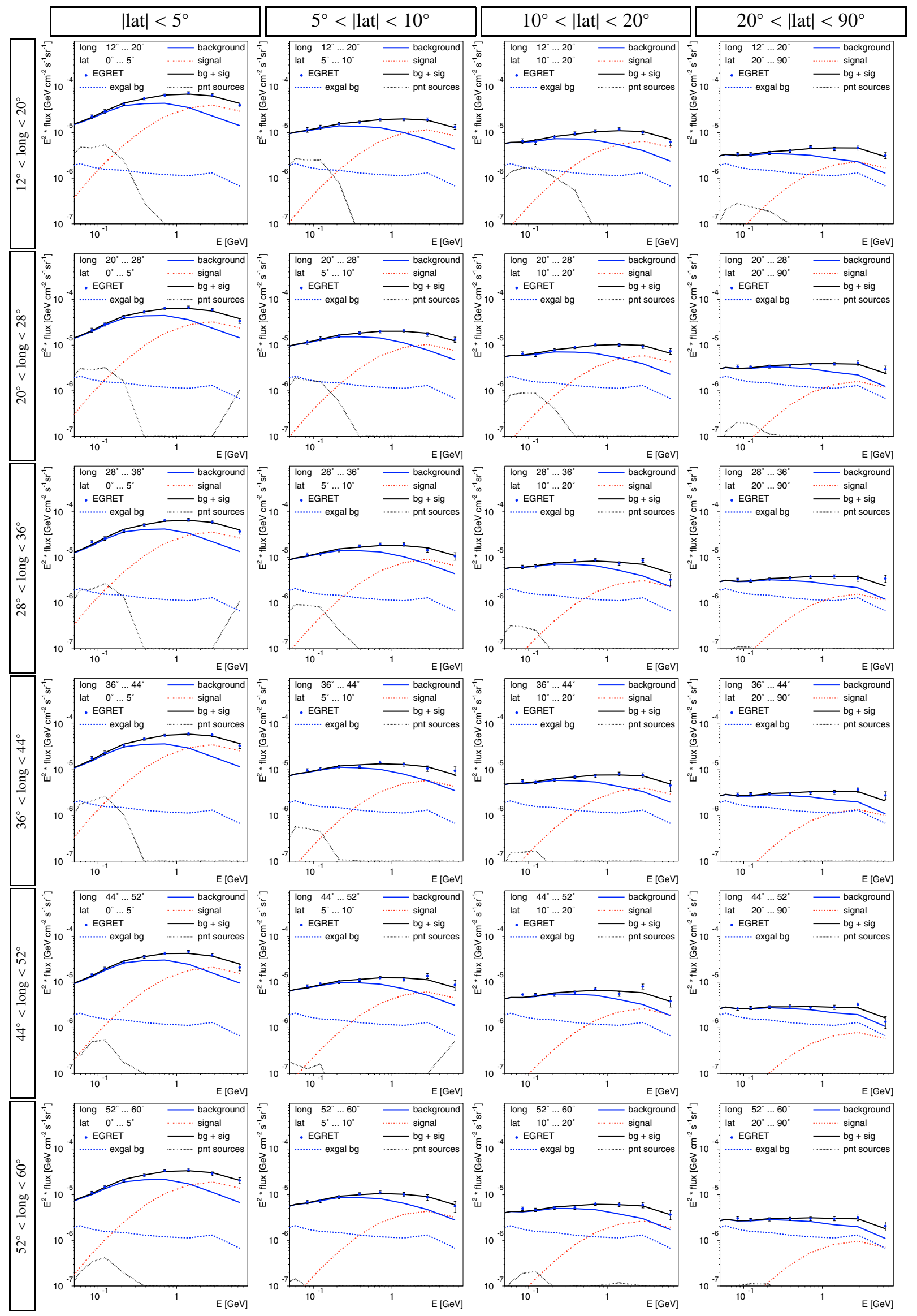


W. de Boer et al.: Diffuse galactic gamma rays as tracer of dark matter, Online Material $p 8$

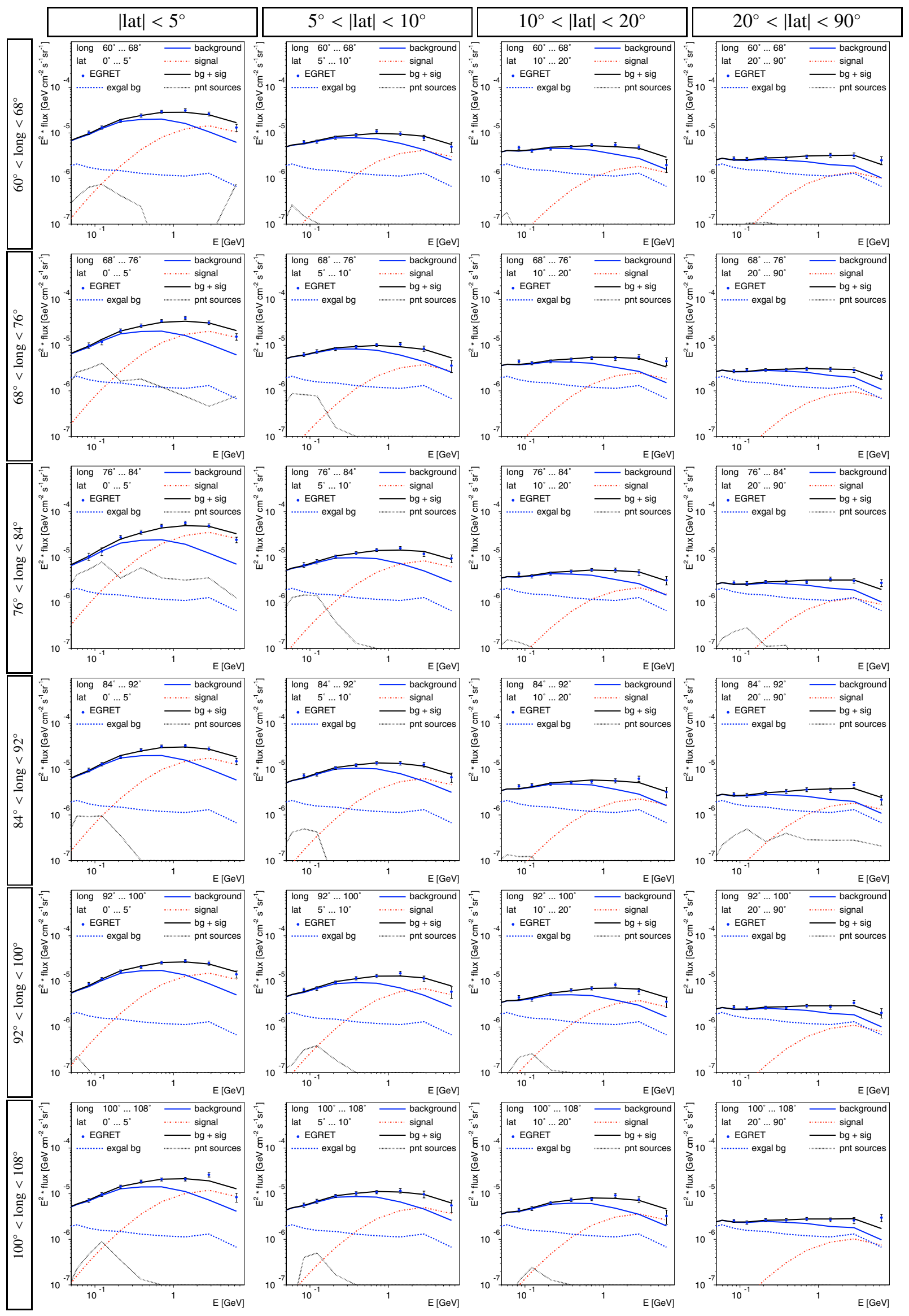


W. de Boer et al.: Diffuse galactic gamma rays as tracer of dark matter, Online Material $p 9$

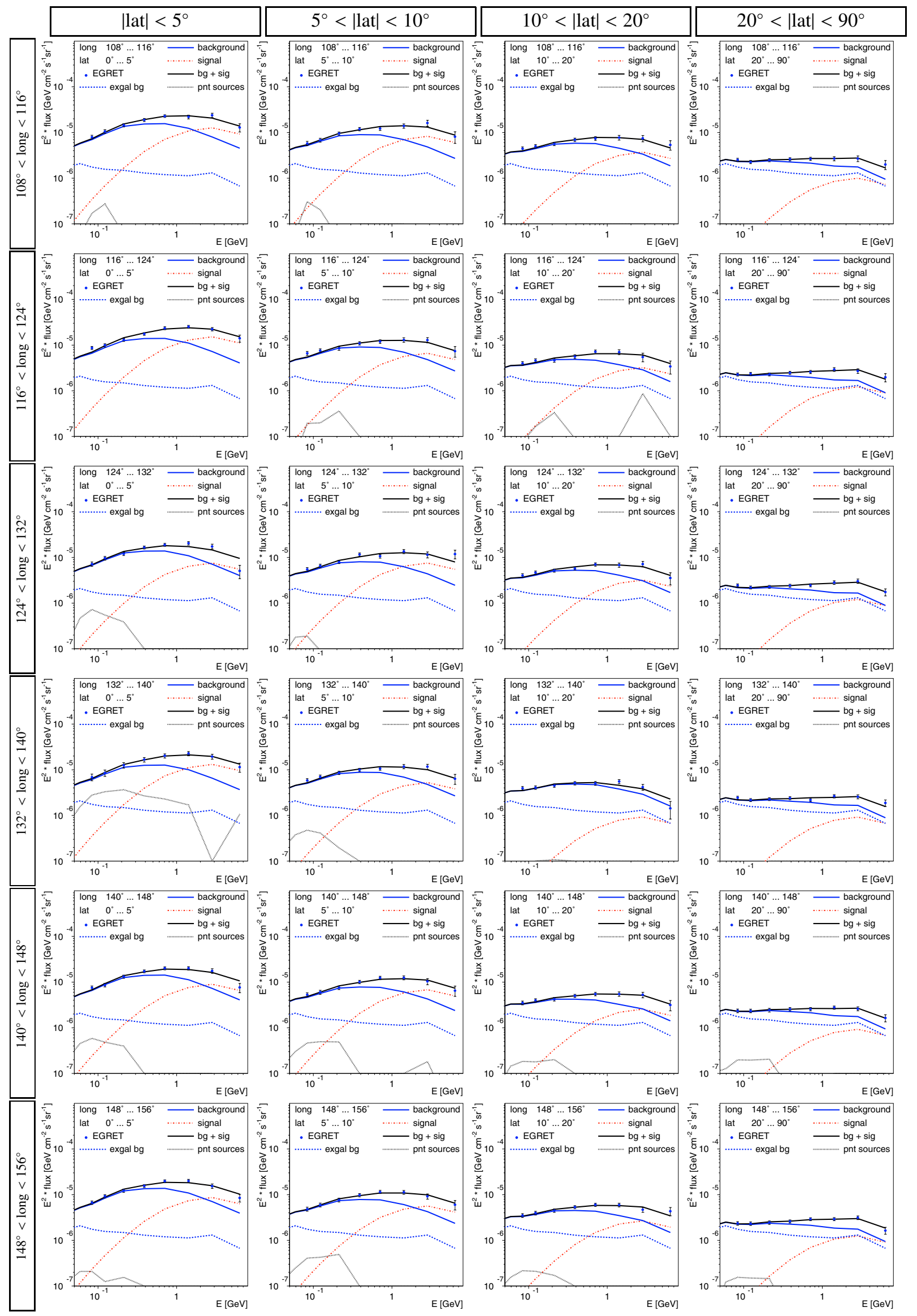


W. de Boer et al.: Diffuse galactic gamma rays as tracer of dark matter, Online Material p 10

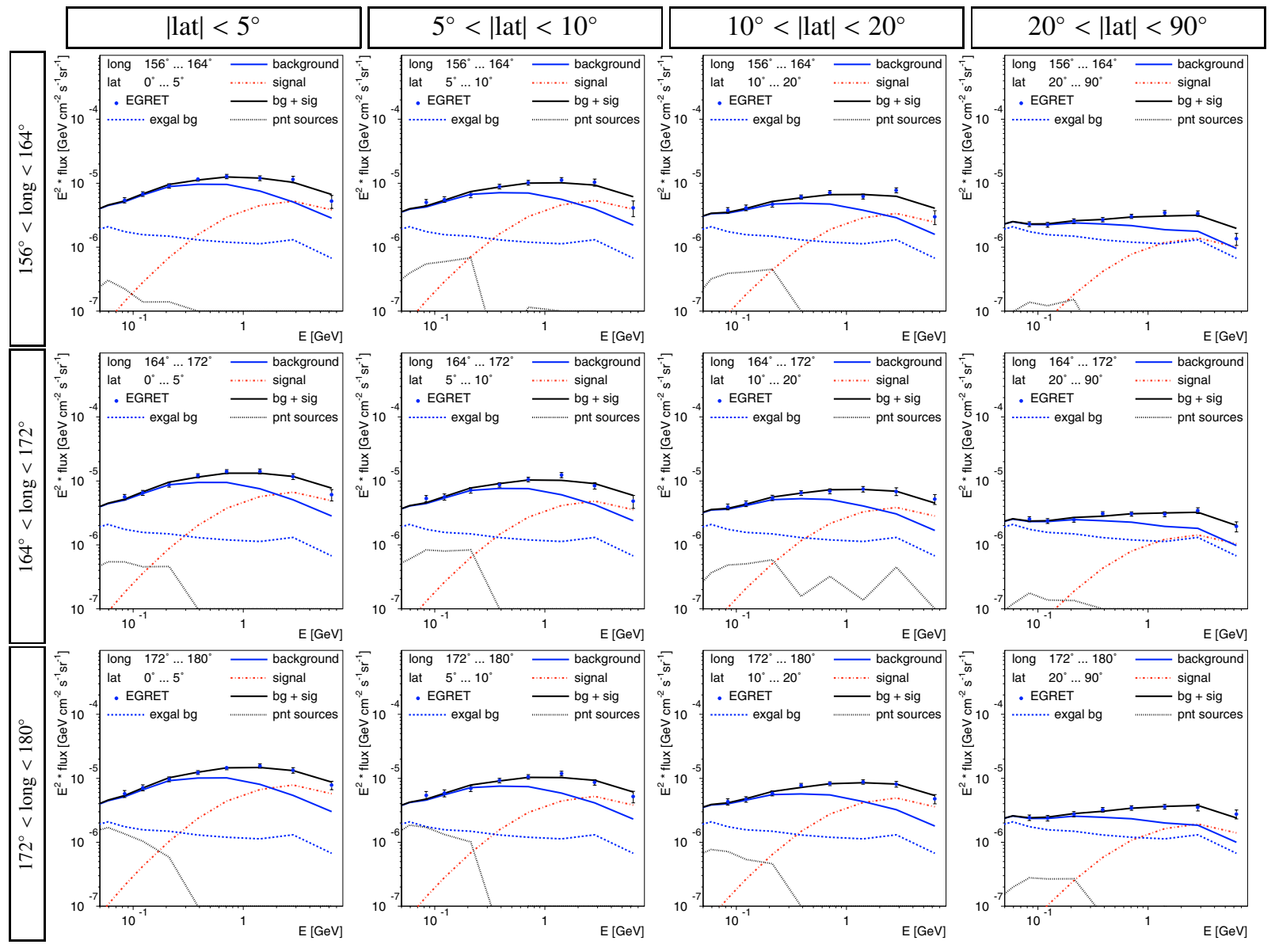

